\title{
A STRATIFICATION OF GENERIC REPRESENTATION THEORY AND GENERALIZED SCHUR ALGEBRAS
}

\author{
NICHOLAS J. KUHN
}

August 2, 2000

\begin{abstract}
If $\mathbf{F}_{q}$ is the finite field of characteristic $p$ and order $q=p^{s}$, let $\mathcal{F}\left(\mathbf{F}_{q}\right)$ be the category whose objects are functors from finite dimensional $\mathbf{F}_{q}$-vector spaces to $\mathbf{F}_{q}$-vector spaces, and with morphisms the natural transformations between such functors.

We define an infinite lattice of thick subcategories of $\mathcal{F}\left(\mathbf{F}_{q}\right)$. Our main result then identifies various subquotients as categories of modules over products of symmetric groups, via recollement diagrams.

Our lattice of thick subcategories is a refinement of the Eilenberg-MacLane polynomial degree filtration $\mathcal{F}^{0}\left(\mathbf{F}_{q}\right) \subset \mathcal{F}^{1}\left(\mathbf{F}_{q}\right) \subset \mathcal{F}^{2}\left(\mathbf{F}_{q}\right) \subset \ldots$ of $\mathcal{F}\left(\mathbf{F}_{q}\right)$ which has been extensively studied and used in the algebraic K-theory literature. Our main theorem implies a description of $\mathcal{F}^{d}\left(\mathbf{F}_{q}\right) / \mathcal{F}^{d-1}\left(\mathbf{F}_{q}\right)$ that refines and extends earlier results of Pirashvili and others.

If $q \geq r$, one of the subcategories is the Friedlander-Suslin category $\mathcal{P}^{r}$ of 'strict polynomial functors of degree r', equivalent to the category of modules over the Schur algebra $S(n, r)$ with $n \geq r$. Our results can thus also be viewed as refining and extending the classic relationship between $S(n, r)$-modules and $\Sigma_{r}$-modules via the Schur functor. In fact, (essentially) all our subcategories are equivalent to categories of modules over various finite dimensional algebras, and our lattice can be interpreted in terms of lattices of idempotent two sided ideals in these generalized Schur algebras.

Applications include a simple proof, free of algebraic group theory, of a generalized Steinberg Tensor Product Theorem. This then implies the classic theorem for $G L_{n}\left(\mathbf{F}_{q}\right)$, shedding some new light on this classic result. Our tensor product theorem is then used to study when various of our generalized Schur algebras are Morita equivalent.

We use two technical tricks which may be of some independent interest. Firstly, we 'twist' by an action of the Galois group $\operatorname{Gal}\left(\mathbf{F}_{q} ; \mathbf{F}_{p}\right)$ to be able to work entirely with vector spaces over the prime field $\mathbf{F}_{p}$, making discussions of 'base change' unnecessary. Secondly, we systematically use 'functors with product', a.k.a. lax symmetric monoidal functors, to define our subcategories.
\end{abstract}

\section{Contents}

\section{Introduction}

1991 Mathematics Subject Classification. Primary 20C30, 20C33, 20G40, Secondary 15A72, 19D55, 20G05.

Partially supported by the N.S.F. 
2. Functors with product and associated functor categories.

2.1. Functors with product

2.2. New algebras from old

2.3. Categories of functors

2.4. Basic structure

2.5. Thick subcategories

2.6. Functors with coproduct and associated comodule categories

\section{Generalized Schur algebras}

4. The categories $F(p, s)$

4.1. Scalar decomposition

4.2. Tensor product

4.3. The Frobenius twist

4.4. Restriction

5. A lattice of functors with coproduct in $F(p, s)$

5.1. Scalar decomposition

5.2. Tensor product

5.3. The Frobenius twist

5.4. Restriction

6. The fine structure of the categories $F^{J}$

7. Simple functors and a Steinberg Tensor Product Theorem

8. The Schur algebras $S(n, J)$

\section{INTRODUCTION}

If $\mathbf{F}_{q}$ is the finite field of characteristic $p$ and order $q=p^{s}$, let $\mathcal{F}\left(\mathbf{F}_{q}\right)$ be the category with objects the functors

$$
F \text { : finite dimensional } \mathbf{F}_{q} \text {-vector spaces } \longrightarrow \mathbf{F}_{q} \text {-vector spaces, }
$$

and with morphisms the natural transformations between such functors. Thus $\mathcal{F}\left(\mathbf{F}_{q}\right)$ is the world in which 'natural' $\mathbf{F}_{q}$-linear algebra is done.

A first observation about this category is that one can view an object $F \in$ $\mathcal{F}\left(\mathbf{F}_{q}\right)$ as a 'generic representation' of the general linear groups over $\mathbf{F}_{q}: F(V)$ becomes an $\mathbf{F}_{q}[G L(V)]$-module for all $\mathbf{F}_{q}$-vector spaces $V$. The tight relationship between $\mathcal{F}\left(\mathbf{F}_{q}\right)$ and the categories of $\mathbf{F}_{q}\left[G L_{n}\left(\mathbf{F}_{q}\right)\right]$-modules, for all $n$, is by now well established [K:I, K:II, K:III, FLS, FS, K3, FFSS], making the study of $\mathcal{F}\left(\mathbf{F}_{q}\right)$ of great representation theoretic interest.

In this paper we define an infinite lattice of localizing subcategories of $\mathcal{F}\left(\mathbf{F}_{q}\right)$, and then identify various subquotients as categories of modules over products of symmetric groups. We do this in a very precise and sharp way. Firstly, by first 'twisting' by an action of the Galois group $\operatorname{Gal}\left(\mathbf{F}_{q} ; \mathbf{F}_{p}\right)$, we replace $\mathcal{F}\left(\mathbf{F}_{q}\right)$ by a more fundamental category $\mathcal{F}(p, s)$ and are thus able to work entirely with vector spaces over the prime field $\mathbf{F}_{p}$. This makes discussions of 'base change' unnecessary, 
and makes transparent discussions of what happens as $q$ varies. Secondly, our subquotient categories are always fit into 'recollement' diagrams (see below).

Our work refines and unifies two previously unrelated results. On one hand, in algebraic $\mathrm{K}$-theoretic contexts, Pirashvili [Pi] and others have investigated the Eilenberg-MacLane polynomial degree filtration of categories of functors such as $\mathcal{F}\left(\mathbf{F}_{q}\right)$. On the other hand, included in our lattices of categories are the FriedlanderSuslin categories [FS] of 'strict polynomial functors of degree r', equivalent to the category of modules over the Schur algebra $S(n, r)$ with $n \geq r$. Here our recollement diagrams refine and extend the classic relationship between $S(n, r)$-modules and $\Sigma_{r}$-modules via the Schur functor. In fact, (essentially) all our subcategories are Morita equivalent to categories of modules over various finite dimensional algebras, and our lattice can be interpreted in terms of lattices of idempotent two sided ideals in these generalized Schur algebras.

We now explain

- the combinatorial definition of our lattice,

- the source of our subcategories,

- the recollement diagrams that result, and

- the construction of associated families of Schur algebras.

(In this introduction, we will ignore our 'Galois twisting' improvement.)

The lattice $\mathcal{L}(p, s)$ is the same one that arose in [K1], and admits the following combinatorial description. Let $\mathbf{N}^{s}$ be the additive monoid of $s$-tuples $J=\left(j_{0}, \ldots, j_{s-1}\right)$ of nonnegative integers. Let $R_{0}, \ldots, R_{s-1} \in \mathbf{Z}^{s}$ be the following vectors: if $s=1, R_{0}=p-1$, and, if $s>1, R_{0}=(-1,0, \ldots, 0, p), R_{1}=$ $(p,-1,0, \ldots, 0), R_{2}=(0, p,-1,0, \ldots, 0), \ldots$, and $R_{s-1}=(0, \ldots, 0, p,-1)$. Let $\mathcal{J}(p, s)$ be the poset $\left(\mathbf{N}^{s}, \leq\right)$, where " $\leq$ " is the partial ordering generated by the inequalities $J<K$, if $K=J+R_{r}$, for some $r$. Then $\mathcal{L}(p, s)=\{\mathcal{J} \subseteq$ $\mathcal{J}(p, s) \mid \mathcal{J}$ is an order ideal $\}$, using the lattice theory terminology [Gra, p.72]: if $\mathcal{K}$ is a poset, $\mathcal{J} \subseteq \mathcal{K}$ is an order ideal if $K \in \mathcal{J}$ and $J \leq K$ implies $J \in \mathcal{J}$.

In [K1], we constructed an explicit bijection between $\mathcal{L}(p, s)$ and the complete lattice of subobjects of the fundamental injective $I_{\mathbf{F}_{q}} \in \mathcal{F}\left(\mathbf{F}_{q}\right)$ defined by $I_{\mathbf{F}_{q}}(V)=$ $\mathbf{F}_{q}^{V^{*}}$. Via duality, this lattice is the opposite of the lattice of quotient objects of the fundamental projective $P_{\mathbf{F}_{q}} \in F\left(\mathbf{F}_{q}\right)$ defined by $P_{\mathbf{F}_{q}}(V)=\mathbf{F}_{q}[V] .{ }^{1}$

We assign a subcategory $\mathcal{F}^{\mathcal{J}} \subseteq \mathcal{F}\left(\mathbf{F}_{q}\right)$ to an order ideal $\mathcal{J} \subseteq \mathcal{J}(p, s)$ in the following way. Let $P^{\mathcal{J}}$ be the associated quotient of the functor $P_{\mathbf{F}_{q}}$. In Lemma 5.4, we will see that $P^{\mathcal{J}}$ admits a natural pairing

$$
P^{\mathcal{J}}(V) \otimes P^{\mathcal{J}}(W) \rightarrow P^{\mathcal{J}}(V \otimes W)
$$

making $P^{\mathcal{J}}$ into a lax symmetric monoidal functor. (We will call this a 'functor with product'.) This allows us to make the following constructions, dual to those in $[\mathrm{K} 2, \S 2.4]$. Let $\mathcal{A}^{\mathcal{J}}$ denote the additive category with objects the finite dimensional $\mathbf{F}_{q}$-vector spaces and with morphisms

$$
\mathcal{A}^{\mathcal{J}}(V, W)=P^{\mathcal{J}}(\operatorname{Hom}(V, W)) .
$$

Then let $\mathcal{F}^{\mathcal{J}}$ be the category of ' $\mathcal{A}^{\mathcal{J}}$-modules': additive functors from $\mathcal{A}^{\mathcal{J}}$ to $\mathbf{F}_{q}$ vector spaces. It is easy to see that $\mathcal{J} \subset \mathcal{K}$ implies that $\mathcal{F}^{\mathcal{J}}$ is a full subcategory of

\footnotetext{
${ }^{1} \mathbf{F}[X]$ denotes the $\mathbf{F}$-vector space with basis the set $X$.
} 
$\mathcal{F}^{\mathcal{K}}$. In Proposition 6.2, we will show that each $\mathcal{F}^{\mathcal{J}} \subseteq \mathcal{F}\left(\mathbf{F}_{q}\right)$ is a thick subcategory, i.e. it is closed under subobjects, quotients, and, least obviously, extensions.

Example 1.1. Given $J=\left(j_{0}, \ldots, j_{s-1}\right) \in \mathcal{J}(p, s)$, let $d(J)=j_{0}+\cdots+j_{s-1}$. Let $\mathcal{J}(p, s, d) \subset \mathcal{J}(p, s)=\{J \mid d(J) \leq d\}$. The corresponding quotient of $P_{\mathbf{F}_{q}}$ is the maximal quotient having Eilenberg-MacLane polynomial degree $\leq d$ and $\mathcal{F}^{\mathcal{J}(p, s, d)}$ can be identified with $\mathcal{F}^{d}\left(\mathbf{F}_{q}\right)$, the category of functors of EilenbergMacLane degree $\leq d .^{2}$

Example 1.2. If $p^{s} \geq r$, let $\mathcal{J}[r] \subset \mathcal{J}(p, s)$ be the order ideal generated by $(r, 0, \ldots, 0) .{ }^{3}$ The resulting category $\mathcal{F} \mathcal{J}[r]$ is the category denoted $\mathcal{P}^{r}$ by E. Friedlander and A. Suslin in [FS]. Moreover, as they observe, $\mathcal{P}^{r}$ is easily seen to be equivalent to the category of modules over the classical Schur algebra $S(n, r)$, for any $n \geq r$.

We need a categorical definition. As in [K:II], a recollement diagram is a diagram of abelian categories

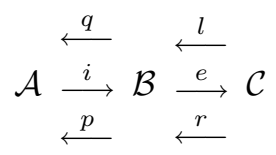

satisfying the following conditions:

- $l$ is left adjoint to $e$, which is left adjoint to $r$. Thus $e$ is exact.

- $q$ is left adjoint to $i$, which is left adjoint to $p$. Thus $i$ is exact.

- $i$ is a full embedding, and $e(B) \simeq 0$ if and only if $B \simeq i(A)$ for some $A$ in $\mathcal{A}$.

- The adjunctions $\epsilon_{C}: \operatorname{er}(C) \rightarrow C$ and $\eta_{C}: C \rightarrow e l(C)$ are isomorphisms for all $C$ in $\mathcal{C}$.

The existance of $e, r, i$ with the cited properties is equivalent to saying that $\mathcal{A}$ can be viewed (via $i$ ) as a thick subcategory of $\mathcal{B}$, and $\mathcal{B} / \mathcal{A} \simeq \mathcal{C}$.

Given a finite order ideal $\mathcal{J} \subset \mathcal{J}(p, s)$, let $\operatorname{Max}(\mathcal{J})$ denote the set of maximal elements of $\mathcal{J}$, and let $\operatorname{Rad}(\mathcal{J})=\mathcal{J}-\operatorname{Max}(\mathcal{J})$. Given $J=\left(j_{0}, \ldots, j_{s-1}\right) \in \mathcal{J}(p, s)$, let $\Sigma_{J}$ be the product of symmetric groups $\Sigma_{j_{0}} \times \cdots \times \Sigma_{j_{s-1}}$. Our main theorem, Theorem 6.10 , is a slightly refined version of the following.

Theorem 1.3. For each finite order ideal, $\mathcal{J} \subset \mathcal{J}(p, s)$, there is a recollement diagram

$$
\mathcal{F}^{\operatorname{Rad}(\mathcal{J})} \underset{i}{\longleftarrow} \mathcal{F}^{\mathcal{J}} \stackrel{\longleftarrow}{\longleftarrow} \prod_{J \in \operatorname{Max}(\mathcal{J})} \mathbf{F}_{q}\left[\Sigma_{J}\right] \text {-modules. }
$$

\footnotetext{
${ }^{2}$ The original reference for polynomial functors in this sense is the 1954 article [EM]. A functor $F$ taking finite dimensional values has degree $\leq d$ if and only if $n \mapsto \operatorname{dim} F\left(\mathbf{F}_{q}^{n}\right)$ is a polynomial function of $n$ of degree $\leq d[\mathrm{~K}: \mathrm{I}]$.

${ }^{3}$ This is independent of the choice of $s$, as soon as $p^{s} \geq r$.
} 
Example 1.4. Corresponding to Example 1.1, we learn that there is a recollement diagram

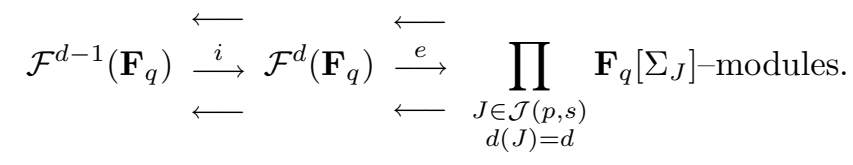

This special case has been known to the author since 1992, and can be viewed as a strengthening for finite fields of a general result of T.Pirashvili [Pi] about polynomial functors on $R$-modules. (See also [Sc, §5.5] for a discussion of a version of the case when $q$ is prime due to Lannes and Schwartz, and dating from 1986.)

Example 1.5. Corresponding to Example 1.2, we learn that there is a recollement diagram

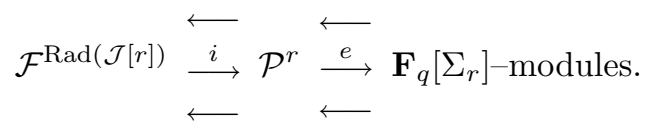

In this last example, the exact functor $e$ is equivalent to the classic Schur functor [Gre, Chapter 6]. More generally, if $J$ is maximal in $\mathcal{J}$, the $J$ th component of $e$,

$$
e^{I}: \mathcal{F}^{\mathcal{J}} \rightarrow \mathbf{F}_{q}\left[\Sigma_{J}\right] \text {-modules, }
$$

is an exact functor that should be regarded as a generalized Schur functor. Furthermore, iterated use of the theorem yields 'secondary' Schur functors defined on the kernels of these primary ones, 'tertiary' functors defined on the kernels of the secondary ones, etc.

Example 1.6. Let $\mathbf{F}=\mathbf{F}_{8}$, so $\mathbf{F}$ has characteristic 2. Then $\mathcal{P}^{6} \subset \mathcal{F}(\mathbf{F})$ corresponds to the order ideal:

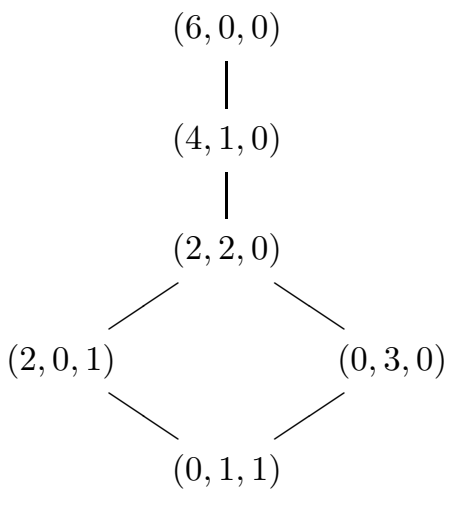

From this, one can thus read off the following. The 'kernel' of the classic Schur functor $\mathcal{P}^{6} \rightarrow \mathbf{F}\left[\Sigma_{6}\right]$-modules will be precisely $\mathcal{P}^{6} \cap \mathcal{F}^{5}(\mathbf{F})$, the degree 5 part of $\mathcal{P}^{6}$. This subcategory then admits an exact functor

$$
\mathcal{P}^{6} \cap \mathcal{F}^{5}(\mathbf{F}) \rightarrow \mathbf{F}\left[\Sigma_{4} \times \Sigma_{1}\right] \text {-modules }
$$


with kernel $\mathcal{P}^{6} \cap \mathcal{F}^{4}(\mathbf{F})$. There is then an exact functor

$$
\mathcal{P}^{6} \cap \mathcal{F}^{4}(\mathbf{F}) \rightarrow \mathbf{F}\left[\Sigma_{2} \times \Sigma_{2}\right] \text {-modules }
$$

with kernel $\mathcal{P}^{6} \cap \mathcal{F}^{3}(\mathbf{F})$. There are then two Schur functors

$$
\mathcal{P}^{6} \cap \mathcal{F}^{3}(\mathbf{F}) \rightarrow \mathbf{F}\left[\Sigma_{2} \times \Sigma_{1}\right] \text {-modules } \times \mathbf{F}\left[\Sigma_{3}\right] \text {-modules }
$$

with kernel $\mathcal{P}^{6} \cap \mathcal{F}^{2}(\mathbf{F})$. Finally, there is an equivalence

$$
\mathcal{P}^{6} \cap \mathcal{F}^{2}(\mathbf{F}) \rightarrow \mathbf{F}\left[\Sigma_{1} \times \Sigma_{1}\right] \text {-modules. }
$$

We do not believe that this sort of stratification has appeared before in the literature.

As was illustrated above in Example 1.2, our categories can often be described as module categories over explicit finite dimensional algebras. If $\mathcal{J} \subseteq \mathcal{J}(p, s)$ is an order ideal, we define an algebra $S(n, \mathcal{J})$, for each $n \geq 0$, by

$$
S(n, \mathcal{J})=P^{\mathcal{J}}\left(M_{n}\left(\mathbf{F}_{q}\right)\right) .
$$

In Theorem 8.5, we show that, if $\mathcal{J}$ is finite, then $\mathcal{F} \mathcal{J}$ is equivalent to the category of $S(n, \mathcal{J})$-modules for all $n \geq n(\mathcal{J})$, where $n(\mathcal{J})$ is explicitly calculated.

The organization of the paper is as follows.

In $\S 2$ and $\S 3$, we describe how lax symmetric monoidal functors yield functor categories and families of generalized Schur algebras. The dual notions - functors with coproduct, coalgebras, etc. - are also discussed.

Using the Galois groups $\operatorname{Gal}\left(\mathbf{F}_{p^{s}}: \mathbf{F}_{p}\right)$, in $\S 4$ we define our 'home' categories $\mathcal{F}(p, s)$ in which we work for the remainder of the paper. $\mathcal{F}(p, s)$ is $\mathbf{F}_{p}$-linear, and yields $\mathcal{F}\left(\mathbf{F}_{p^{s}}\right)$ upon extension of scalars to $\mathbf{F}_{p^{s}}$.

$\S 5$ begins with a review of notation and constructions from [K1]. The subcategories $\mathcal{F}^{J} \subseteq \mathcal{F}(p, s)$ are defined and basic structure discussed.

The heart of the paper is $\S 6$, where our main theorem, Theorem 6.10 , is proved.

The recollement diagrams of the main theorem are used in $\S 7$ to study the simple objects in the categories $\mathcal{F}^{\mathcal{J}}$. In particular, a Steinberg Tensor Product Theorem is easily proved in our setting without any input from the theory of algebraic groups.

In the final section, we define the associated families of Schur algebras $S(n, \mathcal{J})$ and discuss when these are Morita equivalent.

The main results of this paper were announced in the survey paper [K3] written for the Conference on Infinite Length Modules held at the University of Bielefeld, September 1998.

\section{Functors With PRODUCT AND ASSOCIATED FUnCTOR CATEGORIES.}

In this section we elaborate on ideas introduced by us in $[\mathrm{K} 2, \S 2]$. We work over a general field $\mathbf{F}$; thus let $\mathcal{F}(\mathbf{F})$ denote the category whose objects are functors from finite dimensional $\mathbf{F}$-vector spaces to $\mathbf{F}$-vector spaces, and with morphisms the natural transformations between such functors. 
2.1. Functors with product. The category of $\mathbf{F}$-vector spaces is symmetric monoidal using the tensor product as product and $\mathbf{F}$ as unit.

Definition 2.1. A functor $H \in \mathcal{F}(\mathbf{F})$, equipped with the structure of a lax symmetric monoidal functor, is a functor with product.

For the definition of a lax symmetric monoidal functor see [T, M]. Explicitly, a functor with product will be a triple $(H, \mu, \eta)$ consisting of

- a functor $H$ from finite dimensional $\mathbf{F}$-vector spaces to $\mathbf{F}$-vector spaces

- a natural transformation $\mu: H(V) \otimes H(W) \rightarrow H(V \otimes W)$, and

- a linear map $\eta: \mathbf{F} \rightarrow H(\mathbf{F})$

such that the following diagrams commute:

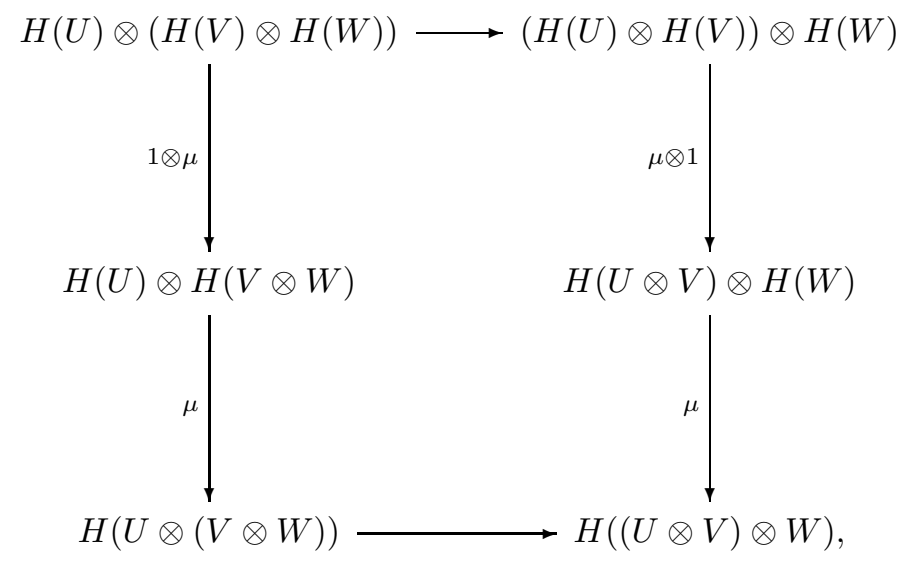

where the horizontal maps are the canonical isomorphisms,

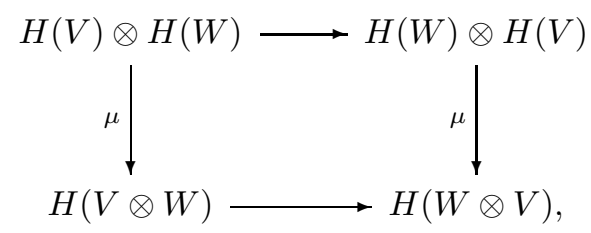

where the horizontal maps are the canonical isomorphisms, and

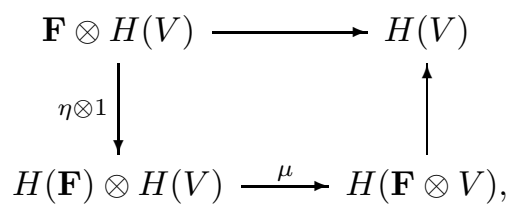

where the unlabelled maps are the canonical isomorphisms.

Example 2.2. Let $P_{\mathbf{F}}$ be defined by $P_{\mathbf{F}}(V)=\mathbf{F}[V]$, the vector space freely generated by the set $V$. This becomes a functor with product by letting the unit be the unit in the group ring $\mathbf{F}[\mathbf{F}]$, and by defining the multiplication as the composite

$$
\mathbf{F}[V] \otimes \mathbf{F}[W]=\mathbf{F}[V \times W] \rightarrow \mathbf{F}[V \otimes W],
$$


where the second map is induced by the canonical bilinear map $V \times W \rightarrow V \otimes W$.

This example is initial among functors with product. Indeed, since $P_{\mathbf{F}}$ satisfies

$$
\operatorname{Hom}_{\mathcal{F}(\mathbf{F})}(P \mathbf{F}, F)=F(\mathbf{F}),
$$

the unit of a functor with product $(H, \mu, \eta)$ corresponds to a unique natural transformation $\tilde{\eta}: P_{\mathbf{F}} \rightarrow H$ easily verified to be a map of functors with product.

Example 2.3. Let $\Gamma^{r}$ be the $r$ th symmetric invariants functor defined by $\Gamma^{r}(V)=$ $\left(V^{\otimes r}\right)^{\Sigma_{r}}$. This has the structure of a functor with product in the obvious way. For example, the multiplication is defined as the inclusion

$$
\Gamma^{r}(V) \otimes \Gamma^{r}(W)=\left(V^{\otimes r} \otimes W^{\otimes r}\right)^{\Sigma_{r} \times \Sigma_{r}} \subseteq\left(V^{\otimes r} \otimes W^{\otimes r}\right)^{\Sigma_{r}}=\Gamma^{r}(V \otimes W) .
$$

2.2. New algebras from old. We refer to a small $\mathbf{F}$-linear category as a multiobject algebra. Explicitly, a multiobject algebra $\mathcal{A}$ has the following structure:

- a set of objects $o b(\mathcal{A})$

- an $\mathbf{F}$-vector space $\mathcal{A}(V, W)$, for all $V, W \in o b(\mathcal{A})$

- unit maps $\eta_{V}: \mathbf{F} \rightarrow \mathcal{A}(V, V)$, for all $V \in o b(\mathcal{A})$

- product maps $\mu_{U, V, W}: \mathcal{A}(V, W) \otimes \mathcal{A}(U, V) \rightarrow \mathcal{A}(U, W)$, for all $U, V, W \in$ $o b(\mathcal{A})$.

These satisfy the usual axioms reflecting the unital and associative properties.

A straightforward check of the definitions proves

Proposition 2.4. (compare with [K2, Prop.2.3]) If $(H, \mu, \eta)$ is a functor with product and $\mathcal{A}$ is a multiobject algebra, then so is $H(\mathcal{A})$, where $H(\mathcal{A})$ has the same objects as $\mathcal{A}, H(\mathcal{A})(V, W)=H(\mathcal{A}(V, W))$, and the structure maps are the composites

$$
\begin{gathered}
H(\mathcal{A}(V, W)) \otimes H(\mathcal{A}(U, V)) \stackrel{\mu}{\longrightarrow} H(\mathcal{A}(V, W) \otimes \mathcal{A}(U, V)) \stackrel{H\left(\mu_{U, V, W}\right)}{\longrightarrow} H(\mathcal{A}(U, W)), \\
\mathbf{F} \stackrel{\eta}{\longrightarrow} H(\mathbf{F}) \stackrel{H\left(\eta_{V}\right)}{\longrightarrow} H(\mathcal{A}(V, V)) .
\end{gathered}
$$

Note that these construction are natural in $\mathcal{A}$ and $(H, \mu, \eta)$.

Example 2.5. Let $H=\Gamma^{r}$ and $\mathcal{A}=M_{n}(\mathbf{F})$, the full matrix ring of $n \times n$ matrices. The resulting new algebra $S(n, r)=\Gamma^{r}\left(M_{n}(\mathbf{F})\right)$ is the classical Schur algebra. Modules over $S(n, r)$ can be interpreted as the degree $r$ polynomial representations of $G L_{n}$, viewed as an algebraic group defined over $\mathbf{F}$.

2.3. Categories of functors. Let $\mathcal{A}(\mathbf{F})$ be the category of finite dimensional $\mathbf{F}$ vector spaces, regarded as a multiobject algebra. Thus $\mathcal{A}(\mathbf{F})(V, W)=\operatorname{Hom}(V, W)$.

Definitions 2.6. Let $H$ be a functor with product.

(1) Let $\mathcal{A}^{H}$ denote the multiobject algebra $H(\mathcal{A}(\mathbf{F}))$. Explicitly, $\mathcal{A}^{H}$ is the $\mathbf{F}$-linear category with objects finite dimensional vector spaces $V$, and with

$$
\mathcal{A}^{H}(V, W)=H(\operatorname{Hom}(V, W)) .
$$


(2) Let $\mathcal{F}^{H}$ be the category of $\mathcal{A}^{H}$-modules, i.e. the category of $\mathbf{F}$-linear functors from $\mathcal{A}^{H}$ to $\mathbf{F}$-vector spaces. Thus an $\mathcal{A}^{H}$-module $M$ will be a collection of vector spaces $M(V)$ together with structure maps

$$
H(\operatorname{Hom}(V, W)) \otimes M(V) \rightarrow M(W)
$$

satisfying various identities.

Example 2.7. Regarding $P_{\mathbf{F}}$ as a functor with product, the resulting algebra $\mathcal{A}^{P_{\mathbf{F}}}$ is the $\mathbf{F}$-linearization of $\mathcal{A}(\mathbf{F})$, and thus the category $\mathcal{F}^{P_{\mathbf{F}}}$ is just the category of functors $\mathcal{F}(\mathbf{F})$.

Since $P_{\mathbf{F}}$ is initial among functors with product, each category $\mathcal{F}^{H}$ has an exact 'forgetful' functor to $\mathcal{F}(\mathbf{F})$. Otherwise put, each category $\mathcal{F}^{H}$ can be regarded as a category of functors.

Example 2.8. Regarding $\Gamma^{r}$ as a functor with product, the resulting category $\mathcal{F}^{\Gamma^{r}}$ is just the Friedlander-Suslin category $\mathcal{P}^{r}$ of functors of scalar (or strict polynomial) degree $r$. (Compare with $[\mathrm{K} 2, \S 3.1]$.)

2.4. Basic structure. Many of the useful constructions in $\mathcal{F}(\mathbf{F})$ (as in [K:I, K:II, $\mathrm{K}$ :III]) carry over to $\mathcal{F}^{H}$. We briefly describe those that we need.

Let $P^{H}(n) \in \mathcal{F}^{H}$ be defined by $P^{H}(n)(V)=H\left(\operatorname{Hom}\left(\mathbf{F}^{n}, V\right)\right)$. These functors are a set of projective generators for $\mathcal{F}^{H}$; indeed these satisfy

$$
\operatorname{Hom}_{\mathcal{F}^{H}}\left(P^{H}(n), M\right)=M\left(\mathbf{F}^{n}\right) .
$$

There is a duality construction, a contravariant functor $D: \mathcal{F}^{H} \rightarrow \mathcal{F}^{H}$. Given $M \in \mathcal{F}^{H}, D M \in \mathcal{F}^{H}$ is defined by letting $D M(V)=M\left(V^{*}\right)^{*}$ with structure map

$$
H(\operatorname{Hom}(V, W)) \rightarrow \operatorname{Hom}(D M(V), D M(W))
$$

defined as the composite of $H(\operatorname{Hom}(V, W)) \rightarrow H\left(\operatorname{Hom}\left(W^{*}, V^{*}\right)\right)$ and

$$
H\left(\operatorname{Hom}\left(W^{*}, V^{*}\right)\right) \rightarrow \operatorname{Hom}\left(M\left(W^{*}\right), M\left(V^{*}\right)\right) \rightarrow \operatorname{Hom}\left(M\left(V^{*}\right)^{*}, M\left(W^{*}\right)^{*}\right) .
$$

It is routine to check that this makes $D M$ into an $\mathcal{A}^{H}$-module. (This uses the symmetry axiom of a lax symmetric monoid functor.) One has

$$
\operatorname{Hom}_{\mathcal{F} H}(M, D N)=\operatorname{Hom}_{\mathcal{F}^{H}}(N, D M)
$$

for all $M, N \in \mathcal{F}^{H}$.

Let $I^{H}(n)=D P^{H}(n) \in \mathcal{F}^{H}$. These functors are a set of injective cogenerators for $\mathcal{F}^{H}$ and satisfy

$$
\operatorname{Hom}_{\mathcal{F}^{H}}\left(M, I^{H}(n)\right)=D M\left(\mathbf{F}^{n}\right) .
$$

Sometimes $\mathcal{F}^{H}$ supports a tensor product with $(M \otimes N)(V)=M(V) \otimes N(V)$. It is easy to see that if $G$ and $H$ are both functors with product, so is $G \otimes H$, and one gets an evident external product $\otimes: \mathcal{F}^{G} \times \mathcal{F}^{H} \rightarrow \mathcal{F}^{G \otimes H}$. Thus a map $H \rightarrow H \otimes H$ of functors with product will yield an internal tensor product on $\mathcal{F}^{H}$.

Example 2.9. The composite $\mathbf{F}[V] \stackrel{\mathbf{F}[\Delta]}{\longrightarrow} \mathbf{F}[V \times V]=\mathbf{F}[V] \otimes \mathbf{F}[V]$ induces the usual tensor product on $\mathcal{F}(\mathbf{F})$. 
Finally, suppose $H$ is 'exponential', i.e. there is a natural isomorphism

$$
H(V \oplus W) \simeq H(V) \otimes H(W)
$$

Then there a map $H \rightarrow H \otimes H$ constructed as in the last example. If this map is a map of functors with product (as seems to often be the case), then one can deduce Kunneth theorems for Ext-groups in $\mathcal{F}^{H}$ as in [K:III]. We leave the details to the interested reader.

2.5. Thick subcategories. Suppose that a map of functors with product $p: G \rightarrow$ $H$ is an epimorphism in $\mathcal{F}(\mathbf{F})$. Via $p$, the abelian category $\mathcal{F}^{H}$ will then be a full subcategory of $F^{G}$ closed under taking subobjects and quotient subobjects. It is interesting to ask when $F^{H}$ is thick, i.e. also closed under extensions.

Lemma 2.10. In this situation, $F^{H}$ is a thick subcategory of $F^{G}$ if and only if, for all $m, n$, we have

$$
\operatorname{Hom}_{\mathcal{F}^{G}}\left(P^{H}(m), I^{G}(n) / I^{H}(n)\right)=0
$$

Proof. $\mathcal{F}^{H}$ is thick if whenever given a short exact sequence in $\mathcal{F}^{G}$

$$
0 \rightarrow M^{\prime} \rightarrow M \rightarrow M^{\prime \prime} \rightarrow 0
$$

with $M^{\prime}, M^{\prime \prime} \in \mathcal{F}^{H}$, then $M \in F^{H}$ as well. By embedding $M^{\prime}$ in an $\mathcal{F}^{H}$-injective and pushing out, then mapping an $\mathcal{F}^{H}$-projective to $M^{\prime \prime}$ and pulling back, we can assume that the sequence has the form

$$
0 \rightarrow I \rightarrow M \rightarrow P \rightarrow 0
$$

where $P$ is an $\mathcal{F}^{H}$-projective and $I$ is an $\mathcal{F}^{H}$-injective. Since such a sequence will split if and only if $M \in \mathcal{F}^{H}$, we wish to know when

$$
\operatorname{Ext}_{F^{G}}^{1}(P, I)=0 \text {. }
$$

Since the family $P^{H}(m)$ are projective generators and $I^{H}(n)$ are injective cogenerators, we see that this will happen for all $P, I$ if and only if

$$
\operatorname{Ext}_{\mathcal{F}^{G}}^{1}\left(P^{H}(m), I^{H}(n)\right)=0
$$

Finally, using the short exact sequence

$$
0 \rightarrow I^{H}(n) \rightarrow I^{G}(n) \rightarrow I^{G}(n) / I^{H}(n) \rightarrow 0
$$

one deduces that

$$
\operatorname{Ext}_{\mathcal{F}^{G}}^{1}\left(P^{H}(m), I^{H}(n)\right)=\operatorname{Hom}_{\mathcal{F}^{G}}\left(P^{H}(m), I^{G}(n) / I^{H}(n)\right) .
$$


2.6. Functors with coproduct and associated comodule categories. Almost everything said so far in this section can be dualized. For example, a triple $(K, \Psi, \epsilon)$ is functor with coproduct if it has the structure obtained by turning all the arrows around in the definition of a functor with product. Such triples (minus the symmetry axiom) were called 'coherent' in [K2, §2.4]. The analogue of Proposition 2.4 was stated as [K2, Prop.2.3].

Let $\mathcal{C}(\mathbf{F})$ denote the multiobject coalgebra dual to $\mathcal{A}(\mathbf{F})$. If $K$ is a functor with product, we let $\mathcal{C}^{K}=K(\mathcal{C}(\mathbf{F}))$. Then the categories of comodules over such $\mathcal{C}^{K}$ 's are the analogues our module categories $\mathcal{F}^{H}$.

Finally, if $H$ is a functor with product that also takes values in finite dimensional vector spaces, then $D H$ is naturally a functor with coproduct, and the categories of $A^{H}$-modules and $\mathcal{C}^{D H}$-comodules will be canonically isomorphic.

Example 2.11. Let $S^{r}$ be the $r$ th symmetric coinvariants functor defined by letting $S^{r}(V)=\left(V^{\otimes r}\right)_{\Sigma_{r}}$. Then $D \Gamma^{r}=S^{r}$, and so the category $\mathcal{P}^{r}$ can be viewed as the category of comodules over the multiobject coalgebra $\mathcal{C}^{S^{r}}$.

Example 2.12. Let $I_{\mathbf{F}}=D P_{\mathbf{F}}$, the fundamental injective in $\mathcal{F}(\mathbf{F})$. If $\mathbf{F}$ is a finite field, then $P_{\mathbf{F}}$ takes finite dimensional values, so that $\mathcal{F}(\mathbf{F})$ can be viewed as the category of comodules over $\mathcal{C}^{I_{\mathbf{F}}}$.

As was already evidenced in [K2], constructions are often more transparent when viewed from the comodule perspective.

\section{Generalized Schur algebras}

In light of Example 2.5, we make the following definition.

Definition 3.1. Let $H$ be a functor with product. Given $n=0,1, \ldots$, let $S(n, H)$ denote the algebra $H\left(M_{n}(\mathbf{F})\right)$.

Let $e(n) \in S(n+1, H)$ be the image of the diagonal matrix with the first $n$ diagonal entries 1 and the last entry 0 under the map

$$
M_{n+1}(\mathbf{F}) \rightarrow \mathbf{F}\left[M_{n+1}(\mathbf{F})\right] \stackrel{\tilde{\eta}}{\rightarrow} H\left(M_{n+1}(\mathbf{F})\right) .
$$

Then $e(n)$ is idempotent, $e(n) S(n+1, H) e(n)$ can be identified with $S(n, H)$, and thus we can define

$$
e_{n+1, n}: S(n+1, H) \text {-modules } \rightarrow S(n, H) \text {-modules }
$$

by the formula $e_{n+1, n}(M)=e(n) M$. The next lemma follows in the usual way. (See $[\mathrm{K}: \mathrm{II}]$.

Proposition 3.2. $e_{n+1, n}$ fits into a recollement diagram

$$
\bar{S}(n+1, H)-\text { modules } \longleftarrow S(n+1, H) \text {-modules } \longleftarrow S(n, H) \text {-modules }
$$

where $\bar{S}(n+1, H)$ is the quotient ring $S(n+1, H) / S(n+1, H) e(n) S(n+1, H)$. 
For any $n \geq 0$, there is also an exact 'evaluation' functor:

$$
e_{n}: \mathcal{F}^{H} \rightarrow S(n, H) \text {-modules }
$$

defined by $e_{n}(M)=M\left(\mathbf{F}^{n}\right)$. These functors are clearly compatible with the functors $e_{n+1, n}$.

Remark 3.3. There is an antiautomorphism of $S(n, H)$ induced by the self map of $M_{n}(\mathbf{F})$ taking a matrix to its transpose. The resulting contravariant functor on the category of $S(n, H)$-modules corresponds, via $e_{n}$, to duality on $\mathcal{F}^{H}$.

The next lemma follows from standard arguments related to Morita equivalence.

Lemma 3.4. For a fixed $H$ and $n$, the following statements are equivalent.

(1) $e_{n}: \mathcal{F}^{H} \rightarrow S(n, H)$-modules is an equivalence.

(2) $e_{m}: \mathcal{F}^{H} \rightarrow S(m, H)$-modules is an equivalence for all $m \geq n$.

(3) $e_{n}(M)=\mathbf{0}$ implies $M=\mathbf{0}$.

(4) $e_{m+1, m}$ is an equivalence for all $m \geq n$.

(5) $\bar{S}(m, H)=0$ for all $m>n$.

(6) $P^{H}(n)$ generates $\mathcal{F}^{H}$.

(7) For each $m>n, P^{H}(m)$ is a quotient of a sum of $P^{H}(n)$ 's.

Example 3.5. We apply the lemma when $H=\Gamma^{r}$ and recover [FS, Theorem 3.2]. The standard isomorphism

$$
\Gamma^{k}(W \oplus V) \simeq \bigoplus_{i+j=k} \Gamma^{i}(W) \otimes \Gamma^{j}(W)
$$

implies that

$$
P^{\Gamma^{r}}(m) \simeq \bigoplus_{r_{1}+\cdots+r_{m}=r} \Gamma^{r_{1}} \otimes \cdots \otimes \Gamma^{r_{m}}
$$

In this sum, at most $r$ of the $r_{i}$ 's in each summand index will be nonzero. Thus $P^{\Gamma^{r}}(m)$ is a quotient of a sum of $P^{\Gamma^{r}}(r)$ 's for all $m$. Criterion (7) of the lemma thus applies with $n=r$. We conclude that, for $n \geq r$, the algebras $S(n, r)$ are all Morita equivalent and the associated module categories are all equivalent to $\mathcal{P}^{r}$.

Example 3.6. Let $H=T^{r}$, the functor defined by $T^{r}(V)=V^{\otimes r}$, with evident structure as a functor with product. There is an isomorphism $P^{T^{r}}(n) \simeq\left(\mathbf{F}^{n}\right)^{\otimes r} \otimes$ $T^{r}$, so that criterion (7) of the lemma applies with $n=1$. We conclude that for all $n \geq 1$ and $r \geq 1$, the algebra $T^{r}\left(M_{n}(\mathbf{F})\right)$ is Morita equivalent to the field $\mathbf{F}$.

Closely related to Lemma 2.10 is the following familiar lemma.

Lemma 3.7. Let $A \rightarrow B$ be an epimorphism of $\mathbf{F}$-algebras with kernel $K$. Then $B$-modules is a thick subcategory of $A$-modules if and only if $K$ is an idempotent ideal.

Proof. Reasoning as in Lemma $2.10, B$-modules will be a thick subcategory of $A$ modules if and only if $\operatorname{Hom}_{A}\left(A / K, \operatorname{Hom}_{\mathbf{F}}(K, \mathbf{F})\right)=0$. But this last vector space is dual to $A / K \otimes_{A} K=K / K^{2}$. 
Corollary 3.8. Let $G \rightarrow H$ be an epimorphism of functors with product and let $K=\operatorname{Ker}\{S(n, G) \rightarrow S(n, H)\}$. Then $S(n, H)$-modules will be a thick subcategory of $S(n, G)-$ modules if and only if $K^{2}=K$.

\section{The Categories $\mathcal{F}(p, s)$}

In this section we introduce our categories $\mathcal{F}(p, s)$. These will be categories defined by means of well chosen functors with product $P_{p^{s}} \in \mathcal{F}\left(\mathbf{F}_{p}\right)$, and having the following desirable properties:

- The $\mathbf{F}_{p^{s}}$ extension of the $\mathbf{F}_{p}$-linear category $\mathcal{F}(p, s)$ is $\mathcal{F}\left(\mathbf{F}_{p^{s}}\right)$.

- All the results about $\mathcal{F}\left(\mathbf{F}_{p^{s}}\right)$ described in the introduction can be obtained by extending the scalars from analogous results about $\mathcal{F}(p, s)$.

Let me explain what I mean by the first property. Let $q=p^{s}$. The values of functors in $\mathcal{F}\left(\mathbf{F}_{q}\right)$ are determined by their values on the vector spaces $\mathbf{F}_{q}^{n}$ and thus on the vector spaces of the form $\mathbf{F}_{q} \otimes V$, where $V$ is an $\mathbf{F}_{p}$-vector space. One way of making sense of the first property is to say that there should be an isomorphism

$$
\mathbf{F}_{q} \otimes P_{q}(V) \simeq P_{\mathbf{F}_{q}}\left(\mathbf{F}_{q} \otimes V\right)
$$

of lax symmetric monoidal functors from $\mathbf{F}_{p}$-vector spaces to $\mathbf{F}_{q}$-vector spaces.

Recalling that $P_{\mathbf{F}_{q}}\left(\mathbf{F}_{q} \otimes V\right)=\mathbf{F}_{q}\left[\mathbf{F}_{q} \otimes V\right]$, one sees that the functor $P_{q}^{\prime}$ defined by

$$
P_{q}^{\prime}(V)=\mathbf{F}_{p}\left[\mathbf{F}_{q} \otimes V\right]
$$

would satisfy this first property. However our second would fail: the interpretation of $\mathcal{F}^{P_{q}^{\prime}}$ would be as the category of functors from $\mathbf{F}_{q}$-vector spaces to $\mathbf{F}_{p}$-vector spaces, and this category would be too small for our purposes, e.g. it has fewer simples than $\mathcal{F}\left(\mathbf{F}_{q}\right)$.

We remedy this problem by using the Galois group $\operatorname{Gal}\left(\mathbf{F}_{q}: \mathbf{F}_{p}\right)$ to 'twist' $P_{q}^{\prime}$.

Definitions 4.1. Let $\operatorname{Gal}\left(\mathbf{F}_{q}: \mathbf{F}_{p}\right)$ act diagonally on $\mathbf{F}_{q}\left[\mathbf{F}_{q} \otimes V\right]: g \bullet \lambda[\mu \otimes v]=$ $g(\lambda)[g(\mu) \otimes v]$ for $g \in \operatorname{Gal}\left(\mathbf{F}_{q}: \mathbf{F}_{p}\right), \lambda, \mu \in \mathbf{F}_{q}$, and $v \in V$.

(1) Define $P_{q}$ to be the functor with product obtained by taking invariants:

$$
P_{q}(V)=\left(\mathbf{F}_{q}\left[\mathbf{F}_{q} \otimes V\right]\right)^{\operatorname{Gal}\left(\mathbf{F}_{q}: \mathbf{F}_{p}\right)} .
$$

(2) Let $I_{q}=D P_{q}$, the dual functor with coproduct.

(3) Let $\mathcal{F}(p, s)=\mathcal{F}^{P^{s}}$, the corresponding category of functors.

This definition may seem rather obscure, but the next lemma reveals that the functor $I_{q}$ has a simpler description.

Lemma 4.2. $I_{q}(V) \simeq S^{*}(V) /\left(v^{q}-v\right)$, the free commutative algebra generated by $V$ modulo the ideal generated by all elements of the form $v^{q}-v$.

This isomorphism in $\mathcal{F}\left(\mathbf{F}_{p}\right)$ of functors with coproduct should be contrasted to the well known fundamental isomorphism in $\mathcal{F}\left(\mathbf{F}_{q}\right)$ [K:I, Lemma 4.12]:

$$
I_{\mathbf{F}_{q}}(W) \simeq S^{*}(W) /\left(w^{q}-w\right) .
$$

This makes it clear that our first desirable property holds. The rest of the paper will make it clear that the second does also. 
The lemma is proved by just carefully keeping track of how the diagonal Galois group action on $P_{\mathbf{F}_{q}}\left(\mathbf{F}_{q} \otimes V\right)$ transports via duality to $I_{\mathbf{F}_{q}}\left(\mathbf{F}_{q} \otimes V\right)$, and then to $\mathbf{F}_{q} \otimes S^{*}(V) /\left(v^{q}-v\right)$ via the isomorphism (4.1).

Constructions in the remainder of the paper generally are easier to describe by using $I_{q}$ and functors with coproduct, than by using $P_{q}$ and functors with product.

Remark 4.3. For those readers who have followed the literature in this area for the past decade, there have been hints that this 'twisting trick' is a good idea. See, e.g. [FLS, Appendix] and [K:III, Remark 2.2].

We now briefly run through some of the basic structure in $\mathcal{F}(p, s)$, analogous to well known structure in $\mathcal{F}\left(\mathbf{F}_{q}\right)$.

4.1. Scalar decomposition. As a functor with coproduct in $\mathcal{F}(p, s), I_{q}$ decomposes into $q$ indecomposable direct summands

$$
I_{q} \simeq I_{q, 0} \oplus I_{q, 1} \oplus \cdots \oplus I_{q, q-1}
$$

where $I_{q, 0}=S^{0}$ is the constant functor, and for $1 \leq r \leq q-1, I_{q, r}$ is the image in $I_{q}$ of $\bigoplus_{k=0}^{\infty} S^{r+k(q-1)}$. Dually, one gets

$$
P_{q} \simeq P_{q, 0} \oplus P_{q, 1} \oplus \cdots \oplus P_{q, q-1}
$$

These then induce a decomposition of abelian categories

$$
\mathcal{F}(p, s) \simeq \mathcal{F}(p, s)_{0} \times \mathcal{F}(p, s)_{1} \times \cdots \times \mathcal{F}(p, s)_{p^{s}-1} .
$$

We say that $M \in \mathcal{F}(p, s)_{r}$ has scalar degree ${ }^{4} r$.

4.2. Tensor product. The evident multiplication $I_{q} \otimes I_{q} \rightarrow I_{q}$ induces a tensor product on $\mathcal{F}(p, s)$.

4.3. The Frobenius twist. The $p$ th power map $\xi: I_{q}(V) \rightarrow I_{q}(V)$ is an automorphism of order $s$ and a map of functors with coproduct. Given $M \in \mathcal{F}(p, s)$, we let $M_{\xi}$ denote the image of $M$ under the induced automorphism of $\mathcal{F}(p, s)$.

4.4. Restriction. The quotient map

$$
I_{p^{s t}}(V) \rightarrow I_{p^{s}}(V)
$$

is a map of functors with coproduct, and it induces the restriction map

$$
\text { res }: \mathcal{F}(p, s t) \rightarrow \mathcal{F}(p, s)
$$

Remark 4.4. This has a two sided exact adjoint ind $: \mathcal{F}(p, s) \rightarrow \mathcal{F}(p, s t)$, with (ind $M)(V)=\operatorname{Hom}_{\mathcal{F}(p, s)}\left(P_{p^{s t}}, M\right)$. However the relationships between these maps and the Frobenius twist (as in [K:II, Prop.5.15]) seem awkward to prove until one extends the scalars to $\mathbf{F}_{p^{s t}}$, and the induction map will not be used elsewhere in this paper.

\footnotetext{
${ }^{4}$ This is called strict polynomial degree in $[\mathrm{FS}]$.
} 


\section{A lattice of Functors With Coproduct in $\mathcal{F}(p, s)$}

In this section we review notation and constructions from [K1], where we determined all the subobjects $I^{\mathcal{J}}$ of the fundamental injective $I_{p^{s}} \in \mathcal{F}(p, s) .{ }^{5}$ To each $I^{\mathcal{J}} \subseteq I_{p^{s}}$, we define a corresponding $\mathcal{F}^{\mathcal{J}} \subseteq \mathcal{F}(p, s)$.

Let $\mathcal{J}(p, s)$ be the poset $\left(\mathbf{N}^{s}, \leq\right)$, where " $\leq$ " is the partial ordering as in the introduction. Recall that this partial order was defined by letting $R_{0}, \ldots, R_{s-1} \in$ $\mathbf{Z}^{s}$ be the following vectors: if $s=1, R_{0}=p-1$, and, if $s>1, R_{0}=$ $(-1,0, \ldots, 0, p), R_{1}=(p,-1,0, \ldots, 0), R_{2}=(0, p,-1,0, \ldots, 0), \ldots$, and $R_{s-1}=$ $(0, \ldots, 0, p,-1)$. Then " $\leq$ " is the partial ordering generated by the inequalities $K>J$, if $K=J+R_{r}$, for some $r$. In [K1, Proposition 3.5], we showed that, if neither $J$ nor $K$ is $\mathbf{0}$, the sequence of all 0 's, then $K \geq J$ if and only if $K-J$ is an $\mathbf{N}$-linear combination of $R_{0}, \ldots, R_{s-1}$.

Let $\mathcal{L}(p, s)=\{\mathcal{J} \subseteq \mathcal{J}(p, s) \mid \mathcal{J}$ is an order ideal $\}$. Thus the elements of $\mathcal{L}(p, s)$ are the downwardly closed subsets of $\mathcal{J}(p, s)$, and $\mathcal{L}(p, s)$ is a distributive lattice using intersection and union as the meet and join operations.

We need to attach various numerical invariants to $J \in \mathcal{J}(p, s)$.

Definitions 5.1. Let $J=\left(j_{0}, \ldots, j_{s-1}\right) \in \mathcal{J}(p, s)$.

(1) Let $r(J)=j_{0}+p j_{1}+\cdots+p^{s-1} j_{s-1}$.

(2) Let $d(J)=j_{0}+j_{1}+\cdots+j_{s-1}$.

(3) Let $\Sigma_{J}$ be the product of symmetric groups $\Sigma_{j_{0}} \times \cdots \times \Sigma_{j_{s-1}}$.

There is a decomposition [K1, Corollary 3.3]

$$
\mathcal{J}(p, s)=\coprod_{r=0}^{p^{s}-1} \mathcal{J}(p, s)_{r}
$$

into indecomposable posets, where $\mathcal{J}(p, s)_{0}=\{\mathbf{0}\}$, and, for $1 \leq r \leq p^{s}-1$, $\mathcal{J}(p, s)_{r}=\left\{J \in \mathcal{J}(p, s)-\{\mathbf{0}\} \mid r(J) \equiv r \bmod p^{s}-1\right\}$. Furthermore, [K1, Corollary 3.8] every proper order ideal $\mathcal{J} \subset \mathcal{J}(p, s)_{r}$ is finite; equivalently, given $J \in \mathcal{J}(p, s)_{r}$, $K>J$ for all but a finite number of $K \in \mathcal{J}(p, s)_{r}$.

As an example, the diagram below shows the lower portion of the infinite poset $\mathcal{J}(2,2)_{1}$. In general, $\mathcal{J}(p, s)_{r}$ would have a diagram that would look roughly like an $s$ dimensional cone.

\footnotetext{
${ }^{5}$ In $[\mathrm{K} 1]$, we studied $I_{\mathbf{F}_{p}^{s}} \in \mathcal{F}\left(\mathbf{F}_{p^{s}}\right)$, but our constructions are defined already in $\mathcal{F}(p, s)$.
} 


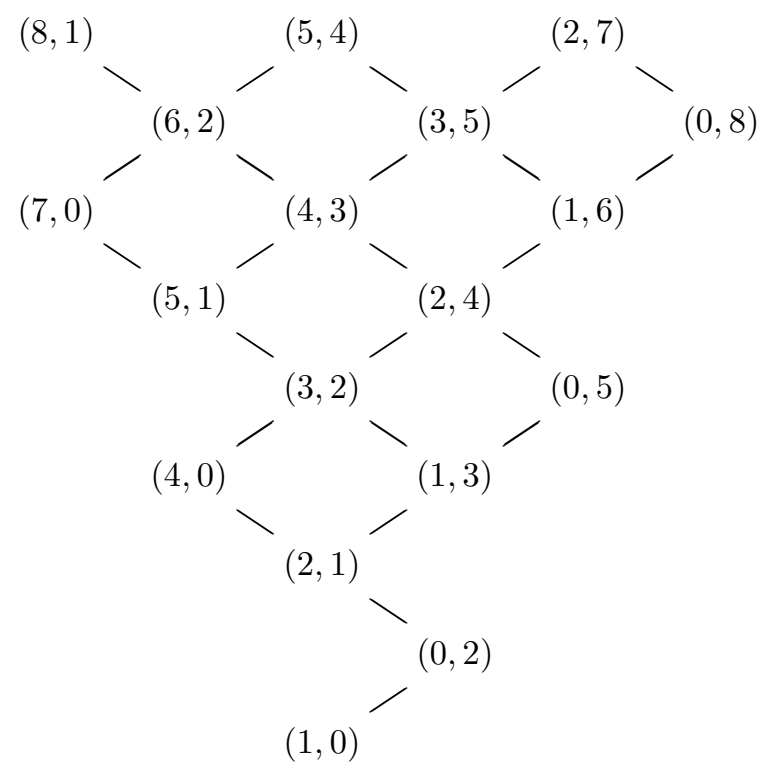

The poset $\mathcal{J}(2,2)_{1}$ in degrees less than 10 .

Now we construct some basic functors in $\mathcal{F}(p, s)$. We start with $T^{j}, S^{j}, \Gamma^{j}, \Lambda^{j}$, and $L^{j}$ defined as follows. $T^{j}(V)=V^{\otimes j}, \Gamma^{j}(V)=\left(V^{\otimes j}\right)^{\Sigma_{j}}$, and $S^{j}(V)=\left(V^{\otimes j}\right)_{\Sigma_{j}}$. $\Lambda^{j}$ is the $j$ th exterior power functor. $L^{j}(V)$ is the degree $j$ component of $L^{*}(V)=$ $S^{*}(V) /\left(v^{p}\right)$, the commutative algebra generated by $V$, modulo the ideal generated by $p$ th powers. Note that $D \Gamma^{j}=S^{j}$, while $T^{j}, \Lambda^{j}$, and $L^{j}$ are all self dual.

Definitions 5.2. Let $J=\left(j_{0}, \ldots, j_{s-1}\right) \in \mathcal{J}(p, s)$.

(1) With $M$ any one of $T, S, \Gamma, \Lambda, L$, let $M^{J}=M^{j_{0}} \otimes M_{\xi}^{j_{1}} \otimes \cdots \otimes M_{\xi^{s-1}}^{j_{s-1}}$.

(2) Let $\Phi^{J}: S^{J} \rightarrow I_{p^{s}}$ be the composite of the maps

$$
\begin{gathered}
S^{j_{0}} \otimes S_{\xi}^{j_{1}} \otimes \cdots \otimes S_{\xi^{s-1}}^{j_{s-1}} \stackrel{p^{t h} \text { powers }}{\longrightarrow} S^{j_{0}} \otimes S^{p j_{1}} \otimes \cdots \otimes S^{p^{s-1} j_{s-1}}, \\
S^{j_{0}} \otimes S^{p j_{1}} \otimes \cdots \otimes S^{p^{s-1} j_{s-1}} \stackrel{\text { multiply }}{\longrightarrow} S^{r(J)},
\end{gathered}
$$

and

$$
S^{r(J)} \stackrel{\pi}{\rightarrow} I_{p^{s}}
$$

where $\pi$ is the inclusion $S^{r(J)} \hookrightarrow S^{*}$ followed by the projection $S^{*} \rightarrow I_{p^{s}}$.

(3) Let $I^{J} \subset I_{p^{s}}$ be the image of $\Phi^{J}$.

We extend the last definition to order ideals.

Definitions 5.3. Let $\mathcal{J} \subseteq \mathcal{J}(p, s)$ be an order ideal.

(1) Let $I^{\mathcal{J}}=\sum_{J \in \mathcal{J}} I^{J} \subseteq I_{p^{s}}$.

(2) Let $P^{\mathcal{J}}=D I^{\mathcal{J}}$.

Lemma 5.4. Each subfunctor $I^{\mathcal{J}} \subseteq I_{p^{s}}$ is a subfunctor with coproduct. 
Proof. By definition, $I^{\mathcal{J}}$ is the image of a map between functors with coproduct. It is easily checked that this map preserves coproduct, as the $p$ th power map and polynomial multiplication maps do.

It follows that each $I^{\mathcal{J}}$ is an object in $\mathcal{F}(p, s)$, and that we can make the following basic definition.

Definition 5.5. If $\mathcal{J} \subseteq \mathcal{J}(p, s)$ is an order ideal, let $\mathcal{F}^{\mathcal{J}} \subseteq \mathcal{F}(p, s)$ be the full subcategory $\mathcal{F}^{P^{\mathcal{J}}}$.

It is easily checked that if $J<K$, then $I^{J} \subset I^{K}$. Thus, if $\mathcal{J} \subseteq \mathcal{K}$, then $I^{\mathcal{J}} \subseteq I^{\mathcal{K}}$ and $\mathcal{F}^{\mathcal{J}} \subseteq \mathcal{F}^{\mathcal{K}}$.

We will need a couple of definitions related to finite order ideals.

Definitions 5.6. Let $\mathcal{J} \subseteq \mathcal{J}(p, s)$ be a finite order ideal.

(1) Let $\operatorname{Max}(\mathcal{J})$ be the set of maximal elements in $\mathcal{J}$.

(2) Let $\operatorname{Rad}(\mathcal{J})$ be the order ideal $\mathcal{J}-\operatorname{Max}(\mathcal{J})$.

Using this terminology we note that

$$
I^{\mathcal{J}} / I^{\operatorname{Rad}(\mathcal{J})} \simeq \bigoplus_{J \in \operatorname{Max}(\mathcal{J})} L^{J}
$$

The main result of [K1] (which we will technically not be using in this paper) is that every every $\mathcal{F}(p, s)$-subobject of $I_{p^{s}}$ is $I^{\mathcal{J}}$ for some order ideal $\mathcal{J}$. It follows from this that $I^{\operatorname{Rad}(\mathcal{J})}=\operatorname{Rad}\left(I^{\mathcal{J}}\right)$, the radical of $I^{\mathcal{J}}$ (viewed as an object in $\mathcal{F}(p, s)$ ).

In the rest of this section, we note how some of the basic structure in the categories $\mathcal{F}(p, s)$, as discussed in the last section, is reflected in the both the posets $\mathcal{J}(p, s)$, and in the categories $\mathcal{F}^{\mathcal{J}}$.

5.1. Scalar decomposition. Any order ideal $\mathcal{J} \subseteq \mathcal{J}(p, s)$ will decompose as a disjoint union of connected order ideals

$$
\mathcal{J}=\coprod_{r=0}^{p^{s}-1} \mathcal{J}_{r}
$$

with $\mathcal{J}_{r}=\mathcal{J} \cap \mathcal{J}(p, s)_{r}$. This corresponds to a decomposition of abelian categories

$$
\mathcal{F}^{\mathcal{J}} \simeq \mathcal{F}_{0}^{\mathcal{J}} \times \mathcal{F}_{1}^{\mathcal{J}} \times \cdots \times \mathcal{F}_{p^{s}-1}^{\mathcal{J}},
$$

with $\mathcal{F}_{r}^{\mathcal{J}}=\mathcal{F}^{\mathcal{J}} \cap \mathcal{F}(p, s)_{r}$.

5.2. Tensor product. Addition give $\mathcal{J}(p, s)$ a monoid structure compatible with the partial ordering, and this induces a product on $\mathcal{L}(p, s)$ : if $\mathcal{J}$ and $\mathcal{K}$ are two order ideals in $\mathcal{J}(p, s)$, then $\mathcal{J} \bullet \mathcal{K}$ is defined to be the smallest order ideal containing the set $\{J+K \mid J \in \mathcal{J}, K \in \mathcal{K}\}$. Then the multiplication $I_{p^{s}} \otimes I_{p^{s}} \rightarrow I_{p^{s}}$ restricts to an epimorphism of functors with coproduct

$$
I^{\mathcal{J}} \otimes I^{\mathcal{K}} \rightarrow I^{\mathcal{J} \bullet \mathcal{K}}
$$

There results a tensor product pairing

$$
\otimes: \mathcal{F}^{\mathcal{J}} \times \mathcal{F}^{\mathcal{K}} \rightarrow \mathcal{F}^{\mathcal{J} \bullet \mathcal{K}}
$$


5.3. The Frobenius twist. Let $\xi: \mathcal{J}(p, s) \rightarrow \mathcal{J}(p, s)$ be the poset automorphism of order $s$ induced by cyclically permuting the factors of $\mathcal{J}(p, s)=\left(\mathbf{N}^{s}, \leq\right)$ : $\xi\left(j_{0}, \ldots, j_{s-1}\right)=\left(j_{s-1}, j_{0}, \ldots, j_{s-2}\right)$. The $p$ th power map $\xi: I_{p^{s}}(V) \rightarrow I_{p^{s}}(V)$ restricts to an isomorphism $\xi: I^{\mathcal{J}} \rightarrow I^{\xi(\mathcal{J})}$ for any order ideal $\mathcal{J} \subseteq \mathcal{J}(p, s)$. Thus $\xi: \mathcal{F}(p, s) \rightarrow \mathcal{F}(p, s)$ restricts to an isomorphism of abelian categories

$$
\xi: \mathcal{F}^{\mathcal{J}} \rightarrow \mathcal{F}^{\xi(\mathcal{J})}
$$

5.4. Restriction. Let res $: \mathcal{J}(p, s t) \rightarrow \mathcal{J}(p, s)$ be the map that sends an $s t-$ tuple $\left(j_{0}, \ldots, j_{s t-1}\right)$ to the $s$-tuple $\left(k_{0}, \ldots, k_{s-1}\right)$ with $k_{v}=\sum_{u \equiv v \bmod s} j_{u}$. It is easy to check that res is order preserving, and that, if $\mathcal{J} \subseteq \mathcal{J}(p, s t)$ is an order ideal, then $\pi\left(I^{\mathcal{J}}\right)=I^{\operatorname{res}(\mathcal{J})}$, where $\pi: I_{p^{s t}} \rightarrow I_{p^{s}}$ is the quotient map. Thus res : $\mathcal{F}(p, s t) \rightarrow \mathcal{F}(p, s)$ restricts to an exact functor

$$
\operatorname{res}: \mathcal{F}^{\mathcal{J}} \rightarrow \mathcal{F}^{\operatorname{res}(\mathcal{J})}
$$

We note that it can easily happen that the surjection res : $\mathcal{J} \rightarrow \operatorname{res}(\mathcal{J})$ is a bijection. In this case res : $\mathcal{F}^{\mathcal{J}} \rightarrow \mathcal{F}^{\text {res }(\mathcal{J})}$ will be an isomorphism.

Example 5.7. Let $\mathcal{J}[r, s] \subset \mathcal{J}(p, s)_{r}$ be the order ideal generated by $(r, 0, \ldots, 0)$. Then $I^{\mathcal{J}[r, s]}$ will be the image of $S^{r}$ in $I_{p^{s}}$. If $p^{s} \geq r$, then this is clearly just $S^{r}$ itself. This corresponds to the combinatorial fact that under the various restriction maps, all the posets $\mathcal{J}[r, s]$ with $p^{s} \geq r$ are isomorphic to each other. Let $\mathcal{J}[r]$ denote any of these; explicitly $\mathcal{J}[r]=\{J \mid r(J)=r\}$. Then $I^{\mathcal{J}[r]}=S^{r}$, and $\mathcal{F} \mathcal{J}[r]$ is the category $\mathcal{P}^{r}$. The identification $\mathcal{J}[a] \bullet \mathcal{J}[b]=\mathcal{J}[a+b]$ corresponds to the tensor product

$$
\otimes: \mathcal{P}^{a} \times \mathcal{P}^{b} \rightarrow \mathcal{P}^{a+b}
$$

and the map of posets $\mathcal{J}[r] \stackrel{\xi}{\rightarrow} \xi(\mathcal{J}[r]) \subset \mathcal{J}[p r]$ corresponds to the exact functor

$$
\Phi: \mathcal{P}^{r} \rightarrow \mathcal{P}^{p r}
$$

that plays a critical role in [FS, FFSS, K2].

Example 5.8. Let $\mathcal{J}(p, s, d) \subset \mathcal{J}(p, s)$ be the order ideal $\{J \mid d(J) \leq d\}$. The corresponding subobject of $I_{p^{s}}$ is the maximal subobject having Eilenberg-MacLane polynomial degree at most $d$, and $\mathcal{F}^{\mathcal{J}(p, s, d)}$ can be identified with $\mathcal{F}^{d}(p, s)$, the category of functors of Eilenberg-MacLane degree $\leq d$. Note that $\mathcal{J}(p, s, d)$ is invariant under $\xi$, and that $\mathcal{J}(p, s, c) \bullet \mathcal{J}(p, s, d)=\mathcal{J}(p, s, c+d)$. Thus $\xi$ restricts to an automorphism

$$
\xi: \mathcal{F}^{d}(p, s) \rightarrow \mathcal{F}^{d}(p, s),
$$

and one gets a tensor product

$$
\otimes: \mathcal{F}^{c}(p, s) \times \mathcal{F}^{d}(p, s) \rightarrow \mathcal{F}^{c+d}(p, s) .
$$




\section{The Fine Structure of the CATEgories $\mathcal{F}^{\mathcal{J}}$}

Throughout this section, we use the following notation: given $M \in \mathcal{F}(p, s)$, $M(n) \in F(p, s)$ is the functor defined by

$$
M(n)(V)=M\left(\operatorname{Hom}\left(\mathbf{F}_{p}^{n}, V\right)\right)=M(\overbrace{V \oplus \cdots \oplus V}^{n}) .
$$

We begin by showing that each category $\mathcal{F}^{\mathcal{J}}$ is a thick subcategory of $\mathcal{F}(p, s)$. Our proof of this uses a fundamental calculation.

Lemma 6.1. ([K1, Lemma 4.2]) Given $J, K \in \mathcal{J}(p, s)$,

$$
\operatorname{Hom}_{\mathcal{F}(p, s)}\left(T^{J}, T^{K}\right) \simeq \begin{cases}\mathbf{F}_{p}\left[\Sigma_{J}\right] \text { as algebras } & \text { if } J=K \\ \mathbf{0} & \text { if } J \neq K\end{cases}
$$

Proposition 6.2. Each category $\mathcal{F}^{\mathcal{J}}$ is a thick subcategory of $\mathcal{F}(p, s)$.

Proof. Using the scalar decomposition, we can assume that $\mathcal{J}$ is connected. If $\mathcal{J}=\mathcal{J}(p, s)_{r}$, then $\mathcal{F}^{\mathcal{J}}$ will just be the subcategory of functors of scalar degree $r$ and is clearly thick. Thus we can assume $\mathcal{J}$ is a proper subset of $\mathcal{J}(p, s)$ and so is finite.

We use Lemma 2.10. To show that $\mathcal{F}^{\mathcal{J}}$ is a thick subcategory, we need to show that, for all $m, n$,

$$
\operatorname{Hom}_{\mathcal{F}(p, s)}\left(P^{\mathcal{J}}(m), I_{p^{s}}(n) / I^{\mathcal{J}}(n)\right)=0
$$

Now $P^{\mathcal{J}}$ has a finite filtration with composition factors $L^{J}, J \in \mathcal{J}$, while $I_{p^{s}} / I^{\mathcal{J}}$ is filtered with composition factors $L^{K}, K \notin \mathcal{J}$. Thus $P^{\mathcal{J}}(m)$ is has a finite filtration with composition factors $L^{J}(m), J \in \mathcal{J}$, while $\left.I_{p^{s}}(n) / I^{\mathcal{J}}(n)\right)$ is filtered with composition factors $L^{K}(n), K \notin \mathcal{J}$. The second statement of the next lemma thus finishes the proof.

\section{Lemma 6.3.}

(1) $\operatorname{Hom}_{\mathcal{F}(p, s)}\left(T^{J}(m), T^{K}(n)\right)=0$ if $J \neq K$.

(2) $\operatorname{Hom}_{\mathcal{F}(p, s)}\left(L^{J}(m), L^{K}(n)\right)=0$ if $J \neq K$.

Proof. Since $L^{J}$ is both a quotient and subobject of $T^{J}$, the Hom-sets of the second statement are subspaces of those in the first. Thus (1) implies (2). Statement (1) follows from Lemma 6.1, and the observation that $T^{J}(n) \simeq n^{d(J)} T^{J}$.

Our goal now is to analyse how $\mathcal{F}^{\operatorname{Rad}(\mathcal{J})}$ and $\mathcal{F}^{\mathcal{J}}$ differ when $\mathcal{J}$ is a finite order ideal, ultimately leading to the analogue in $\mathcal{F}(p, s)$ of Theorem 1.3 of the introduction. The next two propositions include most of what we need.

Proposition 6.4. Let $\mathcal{J}$ be a finite order ideal in $\mathcal{J}(p, s)$, and $M \in \mathcal{F}^{\mathcal{J}}$. Then $M \in \mathcal{F}^{\operatorname{Rad}(\mathcal{J})}$ if and only if, for all $J \in \operatorname{Max}(\mathcal{J}), \operatorname{Hom}_{\mathcal{F}(p, s)}\left(T^{J}, M\right)=0$.

Proposition 6.5. Let $\mathcal{J}$ be a finite order ideal in $\mathcal{J}(p, s)$. If $J$ is a maximal element in $\mathcal{J}$, then $T^{J}$ is both projective and injective in $\mathcal{F}^{J}$.

For the proof of the first of these, we need a lemma. 
Lemma 6.6. Let $\mathcal{J}$ be a finite order ideal in $\mathcal{J}(p, s)$, and $M \in \mathcal{F}^{\mathcal{J}}$. Then $M$ fits into an exact sequence

$$
T \rightarrow M \rightarrow N \rightarrow 0
$$

with $N \in \mathcal{F}^{\operatorname{Rad}(\mathcal{J})}$ and $T$ a direct sum of functors of the form $T^{J}$ with $J \in \operatorname{Max}(\mathcal{J})$.

Proof. Since the functors $P^{\mathcal{J}}(n)$ generate $\mathcal{F}^{\mathcal{J}}$, it suffices to prove the lemma with $M=P^{\mathcal{J}}(n)$. But this case can be done explicitly. There is a short exact sequence

$$
0 \rightarrow \bigoplus_{J \in \operatorname{Max}(\mathcal{J})} L^{J} \rightarrow P^{\mathcal{J}} \rightarrow P^{\operatorname{Rad}(\mathcal{J})} \rightarrow 0
$$

and thus an exact sequence

$$
\bigoplus_{J \in \operatorname{Max}(\mathcal{J})} n^{d(J)} T^{J} \simeq \bigoplus_{J \in \operatorname{Max}(\mathcal{J})} T^{J}(n) \rightarrow P^{\mathcal{J}}(n) \rightarrow P^{\operatorname{Rad}(\mathcal{J})}(n) \rightarrow 0 .
$$

Proof of Proposition 6.4. First suppose that $\operatorname{Hom}_{\mathcal{F}(p, s)}\left(T^{J}, M\right)=0$ for all $J \in$ $\operatorname{Max}(\mathcal{J})$. If we resolve $M$ as in the last lemma,

$$
T \rightarrow M \rightarrow N \rightarrow 0,
$$

we conclude that the first map must be 0 , thus $M \simeq N \in \mathcal{F}^{\operatorname{Rad}(\mathcal{J})}$.

Conversely, suppose $M \in \mathcal{F}^{\operatorname{Rad}(\mathcal{J})}$ and let $J$ be maximal in $\mathcal{J}$. Since $M$ will embed in a product of functors of the form $I^{\operatorname{Rad}(\mathcal{J})}(n)$, so to show that $\operatorname{Hom}_{\mathcal{F}(p, s)}\left(T^{J}, M\right)=0$, it suffices to show that $\operatorname{Hom}_{\mathcal{F}(p, s)}\left(T^{J}, I^{\operatorname{Rad}(\mathcal{J})}(n)\right)=0$ for all $n$. This is then shown as in the proof of Proposition 6.2: $I^{\operatorname{Rad}(\mathcal{J})}$ has a finite filtration with subquotients of the form $L^{K}$ with $K \neq J$, and $L^{K}$ embeds in $T^{K}$, so the result follows from Lemma 6.3(1).

The strategy in proving Proposition 6.5 will be to show that, for a well chosen $n$ that depends on $J \in \operatorname{Max}(\mathcal{J}), T^{J}$ is a direct summand of the $\mathcal{F}^{\mathcal{J}}$-injective $I^{\mathcal{J}}(n)$. Then, since $T^{J}$ is self dual, $T^{J}$ will also be a summand of the $\mathcal{F}^{\mathcal{J}}$-projective $P^{\mathcal{J}}(n)$.

We begin by showing that, very generally, $I^{\mathcal{J}}(n)$ admits a direct sum decomposition. Given $r^{\prime}, r^{\prime \prime} \in \mathbf{N}$, we write $r^{\prime} \equiv r^{\prime \prime}$ if they are both 0 , or if they are both positive and $r^{\prime} \equiv r^{\prime \prime} \bmod p^{s}-1$.

Lemma 6.7. Given $n$ and a finite order ideal $\mathcal{J} \subset \mathcal{J}(p, s)$, there is a commutative diagram

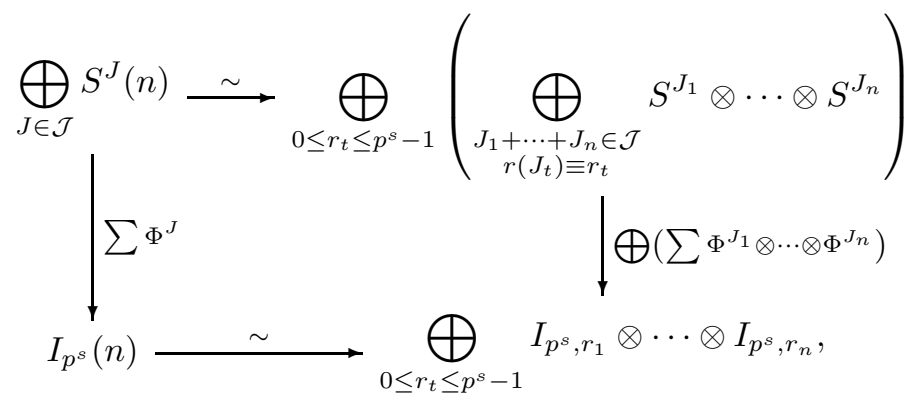


in which the horizontal maps are the isomophisms induced by iterated use of

$$
S^{k}(V \oplus W) \simeq \bigoplus_{i+j=k} S^{i}(V) \otimes S^{j}(V)
$$

and

$$
I_{p^{s}}(V \oplus W) \simeq I_{p^{s}}(V) \otimes I_{p^{s}}(W)
$$

Corollary 6.8. $I^{\mathcal{J}}(n)$ has a natural direct sum decomposition

$$
I^{\mathcal{J}}(n) \simeq \bigoplus_{r_{1}=0}^{p^{s}-1} \cdots \bigoplus_{r_{n}=0}^{p^{s}-1} I_{r_{1}, \ldots, r_{n}}^{\mathcal{J}}
$$

where $I_{r_{1}, \ldots, r_{n}}^{\mathcal{J}} \subset I_{p^{s}}^{\otimes n}$ is the sum ${ }^{6}$ of the tensor products $I^{J_{1}} \otimes \cdots \otimes I^{J_{n}}$ indexed over $n$-tuples $\left(J_{1}, \ldots, J_{n}\right)$ of elements in $\mathcal{J}(p, s)$ such that

$$
J_{1}+\cdots+J_{n} \in \mathcal{J} \text { and also } r\left(J_{1}\right) \equiv r_{1}, \ldots, r\left(J_{n}\right) \equiv r_{n} .
$$

Remark 6.9. In the sum above, it suffices to just consider $\left(J_{1}, \ldots, J_{n}\right)$ such that $J_{1}+\cdots+J_{n} \in \operatorname{Max}(\mathcal{J})$.

Proof of Proposition 6.5. Let $J=\left(j_{0}, \ldots, j_{s-1}\right)$ be maximal in the finite order ideal $\mathcal{J}$. We apply the previous corollary to the case when $n=d(J)$ and

$$
\left(r_{1}, \ldots, r_{n}\right)=(\underbrace{1, \ldots, 1}_{j_{0}}, \underbrace{p, \ldots, p}_{j_{1}}, \ldots, \underbrace{p^{s-1}, \ldots, p^{s-1}}_{j_{s-1}}) .
$$

We claim that the resulting summand is precisely $T^{J}$, thus establishing the proposition.

To see this, for $t=0, \ldots, s-1$, let $J(t) \in \mathcal{J}(p, s)$ be the sequence with a ' 1 ' in the $t$ th place and 0 's elsewhere. Consider the $n$-tuple

$$
\left(J_{1}, \ldots, J_{n}\right)=(\underbrace{J(0), \ldots, J(0)}_{j_{0}}, \underbrace{J(1), \ldots, J(1)}_{j_{1}}, \ldots, \underbrace{J(s-1), \ldots, J(s-1)}_{j_{s-1}}) .
$$

Since $J_{1}+\cdots+J_{n}=J$ and $r(J(t))=p^{t}$, it is in the indexing set of the corollary. Furthermore, $I^{J(t)}=T_{\xi^{t}}^{1}$, so the resulting tensor product $I^{J_{1}} \otimes \cdots \otimes I^{J_{n}}$ is precisely $T^{J}$.

But now we note that this particular $n$-tuple is the only element in the indexing set for the sum. The key to this observation is that $J(t)$ is minimal among all $J$ with $r(J) \equiv p^{t}\left[\mathrm{~K} 1\right.$, Proposition 3.2]. Thus if $\left(K_{1}, \ldots, K_{n}\right)$ satisfies $r\left(K_{1}\right) \equiv$ $r_{1}, \ldots, r\left(K_{n}\right) \equiv r_{n}$, then $K_{i} \geq J_{i}$ for all $i$, and so

$$
K_{1}+\cdots+K_{n} \geq J_{1}+\cdots+J_{n}=J .
$$

If we also impose the condition that $K_{1}+\cdots+K_{n}$ be in the order ideal $\mathcal{J}$, then the fact that $J$ is maximal forces $K_{i}=J_{i}$ for all $i$.

We have arrived at our main theorem, which yields a refined version of Theorem 1.3 of the introduction after extension of scalars.

\footnotetext{
${ }^{6}$ Not necessarily direct.
} 
Theorem 6.10. For each finite order ideal, $\mathcal{J} \subset \mathcal{J}(p, s)$, there is a recollement diagram

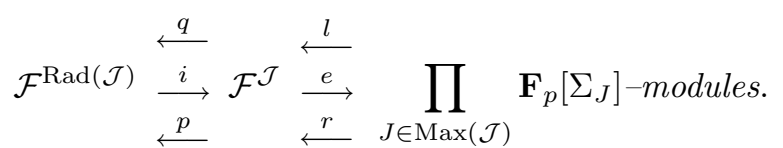

Furthemore,

(1) the Jth component of $e, e^{J}: \mathcal{F}^{\mathcal{J}} \rightarrow \mathbf{F}_{p}\left[\Sigma_{J}\right]$-modules, is given by $e^{J}(F)=$ $\operatorname{Hom}_{\mathcal{F}(p, s)}\left(T^{J}, F\right)$,

(2) the Jth component of $l, l^{J}: \mathbf{F}_{p}\left[\Sigma_{J}\right]$-modules $\rightarrow \mathcal{F}^{\mathcal{J}}$, is given by $l^{J}(M)=$ $\left(T^{J} \otimes M\right)_{\Sigma_{J}}$

(3) the Jth component of $r, r^{J}: \mathbf{F}_{p}\left[\Sigma_{J}\right]$-modules $\rightarrow \mathcal{F}^{\mathcal{J}}$, is given by $r^{J}(M)=$ $\left(T^{J} \otimes M\right)^{\Sigma_{J}}$, and

(4) The canonical natural map $\gamma: l(M) \rightarrow r(M)$ has J th component equal to the $\Sigma_{J}-$ norm map $N_{J}=\sum_{\sigma \in \Sigma_{J}} \sigma:\left(T^{J} \otimes M\right)_{\Sigma_{J}} \rightarrow\left(T^{J} \otimes M\right)^{\Sigma_{J}}$.

Proof. Almost all of this theorem is a formal consequence of Lemma 6.1, Proposition 6.4, and Proposition 6.5. The two propositions show that $\bigoplus_{J \in \operatorname{Max} \mathcal{J}} T^{J}$ is a small ${ }^{7}$ projective generator of the quotient category $\mathcal{F}^{\mathcal{J}} / \mathcal{F}^{\operatorname{Rad}(\mathcal{J})}$, and the lemma computes the endomorphism ring of this generator to be $\prod_{J \in \operatorname{Max}(\mathcal{J})} \mathbf{F}_{p}\left[\Sigma_{J}\right]$. It follows formally that, with $e$ defined as in (1), there is a recollement diagram as claimed, with $l^{J}$ and $r^{J}$ respectively the left and right adjoint to $e^{J}$.

The usual adjunction between $\otimes$ and Hom implies that (2) gives a correct formula for $l^{J}$. It just remains to check formulae (3) and (4).

To verify these 'fine points', we use the lemma below, which asserts that an explicit map induces an isomorphism of $\mathbf{F}_{p}\left[\Sigma_{J}\right]$-modules

$$
e^{J}(D F) \simeq e^{J}(M)^{\#} .
$$

Here, if $M$ is an $\mathbf{F}_{p}\left[\Sigma_{J}\right]$-module, we let $M^{\#}$ denote the dual module. It follows formally then that there is an induced isomorphism

$$
r^{J}\left(M^{\#}\right) \simeq D l^{J}(M) .
$$

Since $D l^{J}(M)=D\left(\left(T^{J} \otimes M\right)_{\Sigma_{J}}\right)=\left(T^{J} \otimes M^{\#}\right)^{\Sigma_{J}}$, we see that formula (3) is true for all injectives, and thus (by resolving modules by injectives) is true in general.

Finally, to check (4), we need to recall [K:II] that

$$
\gamma^{J}: l^{J}(M) \rightarrow r^{J}(M)
$$

is the composite

$$
l^{J}(M) \stackrel{l\left(\epsilon_{2}^{-1}\right)}{\longrightarrow} \operatorname{ler}(M) \stackrel{\epsilon_{1}}{\longrightarrow} r(M),
$$

where $\epsilon_{1}: l e(F) \rightarrow F$ and $\epsilon_{2}: \operatorname{er}(M) \rightarrow M$ are the adjunctions. The first of these

$$
\epsilon_{1}:\left(T^{J} \otimes \operatorname{Hom}_{\mathcal{F}(p, s)}\left(T^{J}, F\right)\right)_{\Sigma_{J}} \rightarrow F
$$

is induced by the evaluation.

\footnotetext{
${ }^{7}$ An object $C \in \mathcal{C}$ is small if $\operatorname{Hom}_{\mathcal{C}}\left(C,{ }_{-}\right)$preserves sums. $T^{J} \in \mathcal{F}(p, s)$ is certainly small: it is even finite.
} 
To get a formula for $\epsilon_{2}^{-1}$, one notes that it is dual to $\eta_{1}^{-1}: e l(M) \rightarrow M$, where $\eta: M \rightarrow e l(M)$ is the adjunction. Under the isomorphism

$$
\left(\mathbf{F}_{p}\left[\Sigma_{J}\right] \otimes M\right)_{\Sigma_{J}} \simeq\left(\operatorname{Hom}_{\mathcal{F}(p, s)}\left(T^{J}, T^{J}\right) \otimes M\right)_{\Sigma_{J}} \rightarrow \operatorname{el}(M)
$$

the map $\eta_{1}^{-1}$ corresponds to the map $\left(\mathbf{F}_{p}\left[\Sigma_{J}\right] \otimes M\right)_{\Sigma_{J}} \rightarrow M$ sending $[\sigma] \otimes m$ to $m$. It follows that, under the isomorphism

$$
\left(\mathbf{F}_{p}\left[\Sigma_{J}\right] \otimes M\right)^{\Sigma_{J}} \simeq\left(\operatorname{Hom}_{\mathcal{F}(p, s)}\left(T^{J}, T^{J}\right) \otimes M\right)^{\Sigma_{J}} \rightarrow \operatorname{er}(M)
$$

the map $\epsilon_{2}^{-1}$ corresponds to the map $M \rightarrow\left(\mathbf{F}_{p}\left[\Sigma_{J}\right] \otimes M\right)^{\Sigma_{J}}$ that sends $m$ to $\sum_{\sigma \in \Sigma_{J}}[\sigma] \otimes m$.

Armed with these explicit formulae for $\epsilon_{1}$ and $\epsilon_{2}^{-1}$, formula (4) is routinely verified.

Lemma 6.11. There is an isomorphism

$$
\operatorname{Hom}_{\mathcal{F}^{J}}\left(T^{J}, D F\right)=\operatorname{Hom}_{\mathcal{F} \mathcal{J}}\left(F, T^{J}\right) \simeq \operatorname{Hom}_{\mathcal{F} \mathcal{J}}\left(T^{J}, F\right)^{\#}
$$

of $\mathbf{F}_{p}\left[\Sigma_{J}\right]$-modules, induced by the pairing

$$
\operatorname{Hom}_{\mathcal{F} \mathcal{J}}\left(F, T^{J}\right) \otimes \operatorname{Hom}_{\mathcal{F} \mathcal{J}}\left(T^{J}, F\right) \stackrel{\text { composition }}{\longrightarrow} \operatorname{Hom}_{\mathcal{F} \mathcal{J}}\left(T^{J}, T^{J}\right)=\mathbf{F}_{p}\left[\Sigma_{J}\right] \stackrel{\delta}{\rightarrow} \mathbf{F}_{p},
$$

where

$$
\delta([\sigma])= \begin{cases}1 & \text { if } \sigma=1 \in \Sigma_{J} \\ 0 & \text { otherwise }\end{cases}
$$

Proof. The pairing is nondegenerate when $F=T^{J}$, so the induced map

$$
\operatorname{Hom}_{\mathcal{F}^{J}}\left(T^{J}, D F\right) \rightarrow \operatorname{Hom}_{\mathcal{F}^{J}}\left(T^{J}, F\right)^{\#}
$$

is an isomorphism in this case. Since both domain and range take direct sums to products in the $F$ variable, this map is also an isomorphism if $F$ is a direct sum of $T^{J}$ 's. Since both domain and range are exact in the $F$ variable, the lemma for an arbitrary $F$ then can be deduced from this, as every $F$ fits into a chain complex

$$
\cdots \rightarrow T_{1} \rightarrow T_{0} \rightarrow F \rightarrow 0
$$

in which each $T_{i}$ is a sum of $T^{J}$ 's and the homology is sent to 0 by $\operatorname{Hom}_{\mathcal{F} \mathcal{J}}\left(T^{J},{ }_{-}\right)$.

Remark 6.12. See the 1995 thesis $[\mathrm{P}]$ for a slightly different presentation of the theorem for $\mathcal{F}(p, 1)$ (filtered by the polynomial degree filtration).

Example 6.13. Recall that $\mathcal{F}^{d}(p, s)$ corresponds to the order ideal $\mathcal{J}(p, s, d)=$ $\{J \mid d(J) \leq d\}$. It follows that there is a recollement diagram

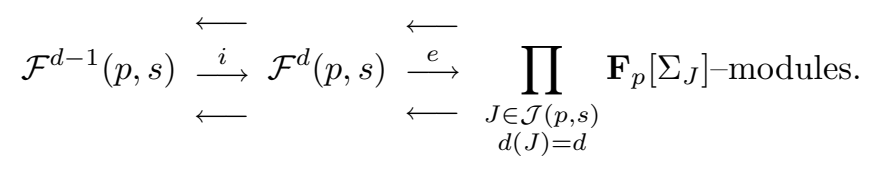


Remark 6.14. Related to this last example, a general result of T.Pirashvili [Pi] about polynomial functors on $R$-modules implies that the quotient category

$$
\mathcal{F}^{d}\left(\mathbf{F}_{p^{s}}\right) / \mathcal{F}^{d-1}\left(\mathbf{F}_{p^{s}}\right)
$$

is equivalent to the modules over the twisted group ring

$$
\left(\mathbf{F}_{p^{s}} \otimes \mathbf{F}_{p} \mathbf{F}_{p^{s}}\right)^{\otimes d}\left[\Sigma_{d}\right]
$$

Using the decomposition of algebras $\mathbf{F}_{p^{s}} \otimes_{\mathbf{F}_{p}} \mathbf{F}_{p^{s}} \simeq \overbrace{\mathbf{F}_{p^{s}} \times \cdots \times \mathbf{F}_{p^{s}}}^{s}$, it is an exercise in Morita theory to show that this ring is Morita equivalent to

$$
\prod_{\substack{J \in \mathcal{J}(p, s) \\ d(J)=d}} \mathbf{F}_{p^{s}}\left[\Sigma_{J}\right] .
$$

\section{Simple functors and a Steinberg Tensor Product Theorem}

In this section we use elementary properties of the symmetric groups together with Theorem 6.10 to learn about the simple objects in $\mathcal{F}(p, s)$. Some of our conclusions were already known [K:II] by applying bits and pieces from the representation theory of the general linear groups. However, our arguments here are truly elementary, and the arguments in [K:II] can be run in reverse to deduce the corresponding results about $G L_{n}\left(\mathbf{F}_{p^{s}}\right)$-modules.

Given $J \in \mathcal{J}(p, s)$, let

$$
c^{J}: \mathbf{F}_{p}\left[\Sigma_{J}\right] \text {-modules } \rightarrow \mathcal{F}(p, s)
$$

be the 'intermediate extension' [K:II] arising from the recollement diagrams of Theorem 6.10. Explicitly,

$$
c^{J}(M)=\operatorname{Im}\left\{N_{J}:\left(T^{J} \otimes M\right)_{\Sigma_{J}} \rightarrow\left(T^{J} \otimes M\right)^{\Sigma_{J}}\right\} .
$$

There is a handy characterization of functors of the form $c^{J}(M)$.

Lemma 7.1. $F \in \mathcal{F}(p, s)$ is isomorphic to $c^{J}(M)$ if and only if $e^{J}(F) \simeq M$, and $F$ is both a quotient of a sum of $T^{J}$ 's and a subobject of a product of $T^{J}$ 's.

Proof. By construction, $c^{J}\left(\mathbf{F}_{p}\left[\Sigma_{j}\right]\right)=T^{J}$. Since $c^{J}$ preserves both epimorphisms and direct sums, it follows that $c^{J}(M)$ will always be a quotient of a sum of $T^{J}$ 's. A similar argument shows that $c^{J}(M)$ always embeds as a subobject of a product of $T^{J}$ 's.

Conversely, if $F$ is such a nice quotient and subobject, then it is in $\mathcal{F}^{\mathcal{J}}$ where $\mathcal{J}$ is the order ideal generated by $J$. Statement (3) of [K:II, Proposition 4.6] then applies, and shows that $F \simeq c^{J}\left(e^{J}(F)\right)$.

We recall a related lemma from the general theory of recollement diagrams.

Lemma 7.2. [K:II, Proposition 4.7] Let $c: \mathcal{C} \rightarrow \mathcal{B}$ be the intermediate extension associated to a recollement diagram

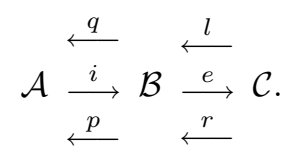


Then there is a bijection between sets of isomorphism classes of simple objects:

$$
\{\text { simples in } \mathcal{A}\} \amalg\{\text { simples in } \mathcal{C}\} \longleftrightarrow\{\text { simples in } \mathcal{B}\}
$$

given by sending a simple $A \in \mathcal{A}$ to $i(A)$ and a simple $C \in \mathcal{C}$ to $c(C)$.

Theorem 6.10 and this lemma together imply

Lemma 7.3. If $\mathcal{J} \subseteq \mathcal{J}(p, s)$ is an order ideal, then a set of isomorphism classes of simple objects in $\mathcal{F}^{\mathcal{J}}$ is given by

$$
\coprod_{J \in \mathcal{J}}\left\{c^{J}(M) \mid M \text { is a simple } \mathbf{F}_{p}\left[\Sigma_{J}\right]-\text { module }\right\} .
$$

Our first application is to splitting fields. A simple object $C$ in an $\mathbf{F}$ linear abelian category $\mathcal{C}$ is called absolutely irreducible if $\operatorname{End}_{\mathcal{C}}(C)=\mathbf{F}$. If every simple is absolutely irreducible, we say that $\mathbf{F}$ is a splitting field for $\mathcal{C}$.

Proposition 7.4. $\mathbf{F}_{p}$ is a splitting field for $\mathcal{F}(p, s)$.

Proof. For any field $\mathbf{F}, \mathbf{F}$ is a splitting field for $\mathbf{F}\left[\Sigma_{j}\right]$-modules. This follows from general representation theory [Se, p.103], since the symmetric groups have the property that if two elements generate the same subgroup, they are conjugate elements.

It is also well known that given finite groups $G$ and $H$, if $\mathbf{F}$ splits both $\mathbf{F}[G]_{-}$ modules and $\mathbf{F}[H]$-modules, then $\mathbf{F}$ splits $\mathbf{F}[G \times H]$-modules [CR, Theorem 10.38]. Thus $\mathbf{F}$ is a splitting field for $\mathbf{F}\left[\Sigma_{J}\right]$-modules for all $J$.

Since intermediate extensions are always full and faithful embeddings, the proposition thus follows from the last lemma.

Remark 7.5. In [K4], we continue and deduce that $\mathbf{F}_{p}$ is a splitting field for the category of modules over the twisted group $\operatorname{ring}\left(\mathbf{F}_{q}\left[G L_{n}\left(\mathbf{F}_{q}\right)\right]\right)^{\operatorname{Gal}\left(\mathbf{F}_{q} ; \mathbf{F}_{p}\right)}$, and thus $\mathbf{F}_{q}$ is a splitting field for $\mathbf{F}_{q}\left[G L_{n}\left(\mathbf{F}_{q}\right)\right]$-modules.

Now we prove a property about duality. (Computationally, this is remarkably fundamental: see, e.g., the crucial way it is used in [K1, §4].)

Proposition 7.6. [K:II, Theorem 7.1] Simple objects in $\mathcal{F}(p, s)$ are self dual.

Proof. Lemma 7.1 shows that $D c^{J}(M)$ is in the image of $c^{J}$ for any $\mathbf{F}_{p}\left[\Sigma_{J}\right]$-module $M$. Since $e^{J}\left(D\left(c^{J}(M)\right)\right) \simeq\left(e^{J}\left(c^{J}(M)\right)\right)^{\#} \simeq M^{\#} \simeq e^{J}\left(c^{J}\left(M^{\#}\right)\right)$, one thus deduces that $D\left(c^{J}(M)\right) \simeq c^{J}\left(M^{\#}\right)$.

General character theory now implies that if $M$ is simple, then it is isomorphic to its dual, as $\Sigma_{J}$ is a group with the property that every element is conjugate to its own inverse.

Remark 7.7. Starting from this proposition, one can then continue and deduce that the transpose dual of a simple $\mathbf{F}_{q}\left[G L_{n}\left(\mathbf{F}_{q}\right)\right]$-module is isomorphic to itself.

Our next goal is to understand in more detail what a simple functor $c^{J}(M)$ looks like. There are a couple of families of simples that are easy to understand. 
Example 7.8. Let $\mathbf{F}_{p}$ denote the trivial $\mathbf{F}_{p}\left[\Sigma_{J}\right]$-module. Then $c^{J}\left(\mathbf{F}_{p}\right)$ will be the image of the norm $N^{J}: T^{J} \rightarrow T^{J}$, easily verified to be the functor $L^{J}$. Let $\epsilon$ denote the one dimensional 'sign' representation of $\mathbf{F}_{p}\left[\Sigma_{J}\right]$. Then $c^{J}(\epsilon)$ will be the image of the signed norm map from $T^{J}$ to $T^{J}$, easily verified to be the functor $\Lambda^{J}$.

Note that these simple functors all decompose as $s$-fold tensor products, with the various factors twisted by the Frobenius. We now show that this is typical.

Let $J=\left(j_{0}, \ldots, j_{s-1}\right)$. Since $\mathbf{F}_{p}$ is a splitting field for $\mathbf{F}_{p}\left[\Sigma_{j}\right]$-modules, it follows that the simple $\mathbf{F}_{p}\left[\Sigma_{J}\right]$-modules are the modules of the form

$$
M_{0} \otimes M_{1} \otimes \cdots \otimes M_{s-1},
$$

where each $M_{t}$ is a simple $\mathbf{F}_{p}\left[\Sigma_{j_{t}}\right]$-module. Furthermore, a simple $\mathbf{F}_{p}\left[\Sigma_{j}\right]$-module will certainly have the form $\mathbf{F}_{p}\left[\Sigma_{j}\right] a$ for some $a \in \mathbf{F}_{p}\left[\Sigma_{j}\right]$.

Lemma 7.9. Let $J=\left(j_{0}, \ldots, j_{s-1}\right)$ and $a_{t} \in \mathbf{F}_{p}\left[\Sigma_{j_{t}}\right]$. If

$$
M \simeq \mathbf{F}_{p}\left[\Sigma_{j_{0}}\right] a_{0} \otimes \mathbf{F}_{p}\left[\Sigma_{j_{1}}\right] a_{1} \otimes \cdots \otimes \mathbf{F}_{p}\left[\Sigma_{j_{s-1}}\right] a_{s-1},
$$

then

$$
c^{J}(M) \simeq T^{j_{0}} a_{0} \otimes\left(T^{j_{1}} a_{1}\right)_{\xi} \otimes \cdots \otimes\left(T^{j_{s-1}} a_{s-1}\right)_{\xi^{s-1}} .
$$

Proof. Let $F=T^{j_{0}} a_{0} \otimes\left(T^{j_{1}} a_{1}\right)_{\xi} \otimes \cdots \otimes\left(T^{j_{s-1}} a_{s-1}\right)_{\xi^{s-1}}$. This functor is both a quotient and subobject of $T^{J}$. Since $e^{J}$ is exact and $e^{J}\left(T^{J}\right)=\mathbf{F}\left[\Sigma_{J}\right]$, we deduce that $e^{J}(F) \simeq M$. By Lemma 7.1, $c^{J}(M) \simeq F$.

Notation 7.10. Let $\Omega$ be an indexing set for the simple $\mathbf{F}_{p}\left[\Sigma_{j}\right]$-modules for all $j$. Given $\omega \in \Omega$, we write $j(\omega)=j$ if $\omega$ corresponds to a simple $\mathbf{F}_{p}\left[\Sigma_{j}\right]-$ module, and, in this situation, we choose $a_{\omega} \in \mathbf{F}_{p}\left[\Sigma_{j}\right]$ so that this simple module is $\mathbf{F}_{p}\left[\Sigma_{j}\right] a_{\omega}$. Then let $F^{\omega}$ be the functor $T^{j} a_{\omega}$. If $\mathcal{J} \subseteq \mathcal{J}(p, s)$ is an order ideal, let

$$
\Omega(\mathcal{J})=\left\{\left(\omega_{0}, \ldots, \omega_{s-1}\right) \in \Omega^{s} \mid\left(j\left(\omega_{0}\right), \ldots, j\left(\omega_{s-1}\right)\right) \in \mathcal{J}\right\} .
$$

With this notation, Lemma 7.3 and Lemma 7.9 combine into a Steinberg Tensor Product Theorem for $\mathcal{F}(p, s)$ and its subcategories $\mathcal{F}^{\mathcal{J}}$.

Theorem 7.11. Let $\mathcal{J} \subseteq \mathcal{J}(p, s)$ be an order ideal. Then a set of isomorphism classes of simple objects in $\mathcal{F}^{\mathcal{J}}$ is given by

$$
\left\{F^{\omega_{0}} \otimes\left(F^{\omega_{1}}\right)_{\xi} \otimes \cdots \otimes\left(F^{\omega_{s-1}}\right)_{\xi^{s-1}} \mid\left(\omega_{0}, \ldots, \omega_{s-1}\right) \in \Omega(\mathcal{J})\right\} .
$$

Notation 7.12. We specialize the notation above. It is well known that $\Omega$ can be taken to be the set of (column) $p$-regular partitions: nondecreasing sequences of nonnegative integers $\omega=(\omega(1), \omega(2), \ldots)$ such that no more than $p-1$ of the positive $\omega(i)$ are equal. With this indexing set $j(\omega)=\sum_{i} \omega(i)$.

G. James [J1, p.41] then gives the following explicit formula for $a_{\omega}$. View the entries of $\omega$ as describing the lengths of the columns in a Young diagram with $j=j(\omega)$ nodes, and let $r_{\omega}, c_{\omega} \in \mathbf{F}_{p}\left[\Sigma_{j}\right]$ respectively be the sum of the elements in the row stabilizer and the signed sum of the elements in the column stabilizer. Then $a_{\omega}=r_{\omega} c_{\omega} r_{\omega}$. 
Example 7.13. Recall that $\mathcal{P}^{r}$ corresponds to the order ideal $\mathcal{J}[r]=\{J \mid r(J)=$ $r$ \} (for any large enough $s$ ). It follows that the simple objects in $\mathcal{P}^{r}$ will be parametrized by the set of all sequences of $p$-regular partitions $\left(\omega_{0}, \ldots, \omega_{s-1}\right)$ such that $\sum_{t} p^{t} j\left(\omega_{t}\right)=r$. It is easy to check that this set corresponds bijectively to the set of all partitions of $r$.

Example 7.14. Recall that $\mathcal{F}^{d}(p, s)$ corresponds to the order ideal $\mathcal{J}(p, s, d)=$ $\{J \mid d(J) \leq d\}$. It follows that the simple objects in $\mathcal{F}^{d}(p, s)$ will be parametrized by the set of all sequences of $p$-regular partitions $\left(\omega_{0}, \ldots, \omega_{s-1}\right)$ such that $\sum_{t} j\left(\omega_{t}\right)=$ $d$.

\section{The Schur algebras $S(n, \mathcal{J})$}

Definition 8.1. Let $\mathcal{J} \subseteq \mathcal{J}(p, s)$ be an order ideal. Define $S(n, \mathcal{J})$ to be the algebra $S\left(n, P^{\mathcal{J}}\right)$, using the notation of $\S 3$. Explicitly,

$$
S(n, \mathcal{J})=P^{\mathcal{J}}\left(M_{n}\left(\mathbf{F}_{p}\right)\right) .
$$

Then let $e(n-1) \in S(n, \mathcal{J})$ be the canonical idempotent so that $e(n-1) S(n, \mathcal{J}) e(n-$ $1)=S(n-1, \mathcal{J})$, and let

$$
\bar{S}(n, \mathcal{J})=S(n, \mathcal{J}) / S(n, \mathcal{J}) e(n-1) S(n, \mathcal{J}) .
$$

It is clear that $\mathcal{J} \subset \mathcal{K}$ implies that there is a quotient map of algebras $S(n, \mathcal{K}) \rightarrow$ $S(n, \mathcal{J})$. Furthermore,

$$
S(n, \mathcal{J}(p, s))=\left(\mathbf{F}_{p^{s}}\left[M_{n}\left(\mathbf{F}_{p^{s}}\right)\right]\right)^{\operatorname{Gal}\left(\mathbf{F}_{p^{s}}: \mathbf{F}_{p}\right)},
$$

so we can view all of our generalized Schur algebras as quotients of these explicit twisted semigroup rings.

Remark 8.2. $\bar{S}(n, \mathcal{J}(p, s))=\left(\mathbf{F}_{p^{s}}\left[G L_{n}\left(\mathbf{F}_{p^{s}}\right)\right]\right)^{\operatorname{Gal}\left(\mathbf{F}_{p^{s}}: \mathbf{F}_{p}\right)}$.

We now use Lemma 3.4, together with our general Steinberg Tensor Product Theorem, to compute for which $n$ the $n$th evaluation functor

$$
e_{n}: \mathcal{F}^{\mathcal{J}} \rightarrow S(n, \mathcal{J}) \text {-modules }
$$

is an equivalence.

Definition 8.3. Given $\omega \in \Omega$, let $n(\omega)=\min \left\{n \mid F^{\omega}\left(\mathbf{F}_{p}^{n}\right) \neq 0\right\}$.

Lemma 8.4. $\max \{n(\omega) \mid j(\omega)=j\}=j$.

Proof. If $j(\omega)=j$, then $F^{\omega}$ is a subfunctor of $T^{j}$. In [K:I, $\left.\S 4\right]$ we gave an elementary proof that if $G$ is any subquotient of $T^{j}$, then $G\left(\mathbf{F}_{p}^{n}\right) \neq 0$. Thus $n(\omega) \leq j$. But this upper bound is realized by the simple functor $\Lambda^{j}$.

Theorem 8.5. Let $\mathcal{J} \subset \mathcal{J}(p, s)$ be a finite order ideal. Then

$$
e_{n}: \mathcal{F}^{\mathcal{J}} \rightarrow S(n, \mathcal{J}) \text {-modules }
$$

is an equivalence if and only if $n \geq n(\mathcal{J})$, where

$$
n(\mathcal{J})=\max \left\{j_{t} \mid\left(j_{0}, \ldots, j_{s-1}\right) \in \mathcal{J}\right\} .
$$


Proof. By criterion (3) of Lemma 3.4, $e_{n}$ will be an equivalence if and only if $F\left(\mathbf{F}_{p}^{n}\right) \neq 0$ for all simple objects $F \in F^{\mathcal{J}}$. Using the description of these simples given in Theorem 7.11, the last lemma shows that the smallest lemma having this property is $n(\mathcal{J})$.

Of course all the equivalent conditions from Lemma 3.4 now apply, e.g. the finite dimensional algebras $S(n, \mathcal{J})$ and $S(m, \mathcal{J})$ will be Morita equivalent if $m$ and $n$ are both at least $n(\mathcal{J})$. Similarly, $\bar{S}(n, \mathcal{J})=0$ if $n>n(\mathcal{J})$.

Remark 8.6. One can refine this theorem using the calculation $n(\omega)=\omega(1)$ (the length of the longest column in the corresponding Young diagram). Using the explicit elements $a_{\omega}=r_{\omega} c_{\omega} r_{\omega}$, it is obvious that $n(\omega) \leq \omega(1)$ : letting $j=j(\omega)$, $F^{\omega}=T^{j} r_{\omega} c_{\omega} r_{\omega}$ is a subquotient of $\Lambda^{\omega}=T^{j} c_{\omega}$. To show that one gets equality, one needs to explicitly find a nonzero element in

$$
\left(\mathbf{F}_{p}^{\omega(1)}\right)^{\otimes j} r_{\omega} c_{\omega} r_{\omega}
$$

This is done in [J2, proof of Thm.3.1].

Suppose that $\mathcal{J} \subset \mathcal{K}$. Then one gets an epimorphism of rings

$$
S(n, \mathcal{K}) \rightarrow S(n, \mathcal{J}),
$$

and an embedding of categories

$$
S(n, \mathcal{J}) \text {-modules } \subset S(n, \mathcal{K}) \text {-modules. }
$$

In the stable range $n \geq \max \{n(\mathcal{J}), n(\mathcal{K})\}$, the subcategory will be thick in the larger one, or equivalently, the kernel of the epimorphism will be an idempotent ideal. However this can easily fail in general.

Example 8.7. Let $p=2, s=1, n=2, \mathcal{J}=(1<2)$, and $\mathcal{K}=(1<2<3<4)$. (Here we view $\mathcal{J}(2,1)$ as the poset $(1<2<3<\ldots)$.) Then $S(2, \mathcal{J})$ is the classic Schur algebra $S(2,2)$, while $S(2, \mathcal{K})$ is the full semigroup ring $\mathbf{F}_{2}\left[M_{2}\left(\mathbf{F}_{2}\right)\right] . \Lambda^{2}\left(\mathbf{F}_{2}^{2}\right)$ is a one dimensional $S(2,2)$-module,

$$
\operatorname{Ext}_{S(2,2)}^{1}\left(\Lambda^{2}\left(\mathbf{F}_{2}^{2}\right), \Lambda^{2}\left(\mathbf{F}_{2}^{2}\right)\right)=0,
$$

but

$$
\operatorname{Ext}_{\mathbf{F}_{2}\left[M_{2}\left(\mathbf{F}_{2}\right)\right]}^{1}\left(\Lambda^{2}\left(\mathbf{F}_{2}^{2}\right), \Lambda^{2}\left(\mathbf{F}_{2}^{2}\right)\right)=\mathbf{F}_{2} .
$$

Thus $S(2, \mathcal{J})$-modules is not a thick subcategory of $S(2, \mathcal{K})$-modules. Note that the stable range here is $n \geq 4$.

This last example reminds us that the classic Schur algebra $S(n, r)$ has finite global dimension [D], and we recall that this is a consequence of $S(n, r)$ being quasi-hereditary, or equivalently that its category of modules is a highest weight category [CPS].

Example 8.8. Let $p=2, s=1$, and $\mathcal{J}=(1<2<3)$. Then $\mathcal{F}^{\mathcal{J}}=\mathcal{F}^{3}\left(\mathbf{F}_{2}\right)$, the category of functors of degree $\leq 3$ in $\mathcal{F}\left(\mathbf{F}_{2}\right)$. It is not hard to explicitly compute that the algebra $\operatorname{Ext}_{\mathcal{F}^{3}\left(\mathbf{F}_{2}\right)}^{*}\left(\Lambda^{3}, \Lambda^{3}\right)$ is isomorphic to a polynomial algebra on an element in $\operatorname{Ext}^{2}$. Thus neither $\mathcal{F}^{\mathcal{J}}$ nor any of the Schur algebras $S(n, \mathcal{J})$ with $n \geq 3$ have finite global dimension. 
Question 8.9. For what finite order ideals $\mathcal{J} \subset \mathcal{J}(p, s)$ does the category $\mathcal{F} \mathcal{J}$ have finite global dimension?

We remark that $\mathcal{F}^{\mathcal{J}}$ having finite global dimension is presumably equivalent to the injective object $I^{\mathcal{J}}$ having a projective resolution in $\mathcal{F}^{\mathcal{J}}$ of finite length.

\section{REFERENCES}

[CR] C. W. Curtis and I. Reiner, Methods of Representation Theory: Vol.I, John Wiley and Sons, New York, 1981.

[CPS] E. Cline, B. J. Parshall, and L. L. Scott, Finite dimensional algebras and highest weight categories, J. reine angew. Math. 391 (1988), 85-99.

[D] S. Donkin, On Schur algebras and related algebras I, J. Algebra. 104 (1986), 310-328.

[EM] S. Eilenberg and S. MacLane, On the groups $H(\pi, n)$, II, Ann. Math. 60 (1954), 49-139.

[FFSS] V. Franjou, E. M. Friedlander, A. Skorichenko, and A. Suslin, General linear and functor cohomology over finite fields, Ann. Math. 150(1999), 663-728.

[FLS] V. Franjou, J. Lannes, and L. Schwartz, Autour de la cohomologie de MacLane des corps finis, Invent. Math. 115(1994), 513-538.

[FS] E. M. Friedlander and A. Suslin Cohomology of finite group schemes over a field, Inv. Math. 127(1997), 209-270.

[Gra] G. Grätzer, Lattice Theory: First Concepts and Distributive Lattices, W. H. Freeman and Co., San Francisco, 1971.

[Gre] J. A. Green, Polynomial Representations of $G L_{n}$, Springer L. N. Math. 830, New York, 1980.

[J1] G. D. James, The representation theory of the symmetric groups, Springer L. N. Math. 682, New York, 1978.

[J2] G. D. James, The decomposition of tensors over fields of prime characteristic, Math. Ziet. 172(1980), 161-178.

[K:I] N. J. Kuhn, Generic representation theory of the finite general linear groups and the Steenrod algebra: I, Amer. J. Math. 116(1994), 327-360.

[K:II] N. J. Kuhn, Generic representation theory of the finite general linear groups and the Steenrod algebra: II, K-Theory J. 8(1994), 395-428.

[K:III] N. J. Kuhn, Generic representation theory of the finite general linear groups and the Steenrod algebra: III, K-Theory J. 9(1995), 273-303.

[K1] N. J. Kuhn, Invariant subspaces of the ring of functions on a vector space over a finite field, J. Algebra 191(1997), 212-227.

[K2] N. J. Kuhn, Rational cohomology and cohomological stability in generic representation theory, Amer. J. Math.120(1998), 1317-1341.

[K3] N. J. Kuhn, The generic representation theory of finite fields: a survey of basic structure, to appear in the Proceedings of the 1998 Bielefeld University Conference on Infinite Length Modules, Trends in Mathematics, Birkhauser.

[K4] N. J. Kuhn, Splitting fields and twisted group rings for the finite general linear groups, to appear in the Proceedings of the 1998 University of Virginia Conference on Modular Representation Theory, de Gruyter.

[M] J. P. May, Brave new worlds in stable homotopy theory, Homotopy theory via algebraic geometry and group representations, Evanston, 1997, A.M.S. Cont. Math. 220(1998), 193212.

[Pi] T. Pirashvili, Higher additivisations [Russian, English summary], Trudy Tbiliss. Mat. Inst. Razmodze Akad. Nauk Gruzin. SSR 91(1988), 44-54.

[P] L. Piriou, Extensions entre foncteurs de la catégorie des espaces vectoriels sur le corps premier à $p$ éléments dans elle-même, thèse de doctorat, Université Paris VII, 1995.

[Sc] L. Schwartz, Unstable modules over the Steenrod algebra and Sullivan's fixed point conjecture, Chicago Lectures in Math., University of Chicago Press, 1994.

[Se] J.-P. Serre, Linear Representations of Finite Groups, Graduate Texts in Mathematics Vol.42, Springer-Verlag, New York, 1977.

[T] R. W. Thomason, First quadrant spectral sequences in algebraic K-theory, Algebraic Topology, Aarhus 1978, Springer L.N.Math 763(1979), 332-355. 
Department of Mathematics, University of Virginia, Charlottesville, VA 22903 